Charité Centrum für Magen-, Darm-, Nieren- und Stoffwechselmedizin Medizinische Klinik für

Gastroenterologie, Infektiologie und Rheumatologie, Campus Benjamin Franklin (Leiterin: Prof. Dr. Britta Siegmund)

\title{
Habilitationsschrift
}

\section{Therapie der axialen Spondyloarthritis}

zur Erlangung der venia legendi

für das Fach

Innere Medizin

Vorgelegt dem Fakultätsrat der Medizinischen Fakultät

Charité - Universitätsmedizin Berlin

von

Dr. med. Hildrun Haibel

Eingereicht: Oktober 2014

Dekanin: $\quad$ Prof. Dr. med. Annette Grüters-Kieslich

1. Gutachter: Prof. Dr. med. Hendrik Schulze-Koops

2. Gutachter: $\quad$ Prof. Dr. med. Bernhard Manger 
Der vorliegenden kumulativen Habilitationsschrift liegen folgende Arbeiten zugrunde:

1. Haibel H, Fendler C, Listing J, Callhoff J, Kraus A, Braun J, Sieper J. Efficacy of oral prednisolone in active ankylosing spondylitis - Results of a double blind placebo controlled short term trial. Ann Rheum Dis. 2014 Jan; 73(1):243-6.

2. Haibel H, Heldmann F, Listing J, Kupper H, Braun J, Sieper J. Long term efficacy of adalimumab after drug withdrawal and retreatment of flare patients in active nonradiographic axial spondyloarthritis. Arthritis Rheum2013 Aug;65(8):2211-3

3. Haibel H, Rudwaleit M, Listing J, Heldmann F, Wong RL, Kupper H, Braun J, Sieper J. Efficacy of Adalimumab in the Treatment of Axial Spondyloarthritis Without Radiographically Defined Sacroiliitis, Arthritis Rheum. 2008 Jul;58(7):1981-91

4. Haibel H, Song IH, Rudwaleit M, Listing J, Hildeman S, Sieper J, Multicenter open label study with infliximab in active ankylosing spondylitis over 28 weeks in daily practice. Clin Exp Rheumatol. 2008 Mar-Apr; 26(2):247-52.

5. Haibel $\mathbf{H}$, Brandt $H C$, Song IH, Brandt A, Listing J, Rudwaleit M, Sieper J. No efficacy of subcutaneous methotrexate in active ankylosing spondylitis - a 16 weeks open label trial. Ann Rheum Dis. 2006 Aug 10; Ann Rheum Dis. 2007 Mar; 66(3):419-21.

6. Haibel H, Niewerth M, Brandt J, Rudwaleit M, Listing J, Sieper J, Braun J. Measurement of quality of life in patients with active ankylosing spondylitis being treated with infliximab- a comparison of SF-36 and SF-12, Z Rheumatol. 2004 Oct;63(5):393-401. German.

7. Haibel H, Brandt J, Rudwaleit M, Soerensen H, Sieper J, Braun J. Treatment of active ankylosing spondylitis with pamidronate. Rheumatology (Oxford). 2003 Aug; 42(8):1018-20. 


\section{Inhaltsverzeichnis}

$\begin{array}{lll}\text { 1. } & \text { Einleitung } & 6\end{array}$

$\begin{array}{lll}\text { 1.1. Ankylosierende Spondylitis } & 6\end{array}$

1.1.1. Diagnose der ankylosierenden Spondylitis $\quad 8$

1.1.2. Therapie der ankylosierenden Spondylitis $\quad 8$

1.2. Nicht-röntgenologische axiale Spondyloarthritis 13

1.2.1. Diagnose der nicht-röntgenologischen axialen Spondyloarthritis 13

1.2.2. Therapie der nicht-röntgenologischen axialen Spondyloarthritis 15

$\begin{array}{lll}2 . & \text { Ergebnisse } & 17\end{array}$

2.1. Outcomeparameter bei der ankylosierenden Spondylitis 17

2.1.1. Evaluation des Lebensqualitätsfragebogens SF-12 als Outcome Parameter bei der ankylosierenden Spondylitis im deutschen Sprachraum

2.2. Alternative Therapieansätze der ankylosierenden Spondylitis 28

2.2.1. Pamidronat bei der ankylosierenden Spondylitis 28

2.2.2. Methotrexat bei der ankylosierenden Spondylitis 34

2.2.3. Steroide bei der Therapie der ankylosierenden Spondylitis am Beispiel 39
von Prednisolon

2.3. TNF-alpha Blocker in der Therapie der ankylosierenden Spondylitis $\quad 45$

2.3.1. Effektivität von Infliximab in der Routineversorgung bei Patienten mit 45

2.4. Therapie der nicht- röntgenologischen axialen Spondyloarthritis 54

2.4.1 Adalimumab bei nicht-röntgenologischer axialer Spondyloarthritis 54

2.4.2. Langzeiteffekt von Adalimumab bei
röntgenologischer axialer Spondyloarthritis

3 Diskussion $\quad 73$

$4 \quad$ Zusammenfassung $\quad 79$

$\begin{array}{lll}5 & \text { Literaturverzeichnis } & \mathbf{8 2}\end{array}$ 
6

Danksagung

$7 \quad$ Erklärung 


\section{Abkürzungen}

AS

ASAS

ASDAS

BASDAI

BASFI

BASMI

BSG

CRP

DMARD

DXA

ESSG

EULAR

HLA-B27

i.v.

KG

MRT

MTX

nr-axSpA

NRS

NSAR

p.o.

RA

RCT

SF-12

SF-36

SIG

SpA

STIR

TNF-alpha

VAS

WHO

WPAI

WS

z.B.
Ankylosierende Spondylitis

Assessment of SpondyloArthritis international Society

Ankylosing Spondylitis Disease Activity Score

Bath Ankylosing Spondylitis Disease Activity Index

Bath Ankylosing Spondylitis Functional Index

Bath Ankylosing Spondylitis Metrology Index

Blutsenkungsgeschwindigkeit

C-reaktives Protein

Disease Modifying Antirheumatic Drug

dual-energy X-ray absorptiometry

European Spondyloarthritis Study Group

European League Against Rheumatism

Humanes Leukozyten Antigen-B27

intravenös

Körpergewicht

Magnetresonanztomographie

Methotrexat

nicht-röntgenologische axiale Spondyloarthritis

Numeric Rating Scale

Nicht- Steroidale Antirheumatika

per os

Rheumatoide Arthritis

Randomized Controlled Trial

Short-Form 12 (Fragebogen zur Erfassung der Lebensqualität)

Short-Form 36 (Fragebogen zur Erfassung der Lebensqualität)

Sakroiliakalgelenk

Spondyloarthritis

Short Tau Inversion Recovery

Tumor Nekrose Faktor alpha

Visual Analog Scale

World Health Organization

Work Productivity and Activity Impairment Questionnaire

Wirbelsäule

zum Beispiel 


\section{Einleitung}

\subsection{Ankylosierende Spondylitis}

\subsubsection{Diagnose der ankylosierenden Spondylitis}

In der Gruppe der Spondyloarthritiden (SpA) ist die ankylosierende Spondylitis (AS) eine häufige entzündlich- rheumatische Erkrankung und Prototyp mit einer Prävalenz von ca. $0.5 \%[1,2]$. Es findet sich vorwiegend eine Entzündung und mögliche folgende Versteifung der Sakroiliakalgelenke (SIG) und der Wirbelsäule (WS). Überwiegend sind junge Erwachsene im arbeitsfähigen Alter betroffen [3]. Es besteht eine hohe Assoziation mit dem Gen HLA-B27, 80-95\% der Patienten sind positiv [1]. Die Diagnose der AS wird nach den modifizierten New York Kriterien von 1984 gestellt (Abbildung 1) und umfasst neben den klinischen Kriterien Morgensteifigkeit, Bewegungseinschränkungen der Wirbelsäule und/oder des Thorax auch das radiologische Kriterium einer definitiven Sakroiliits Grad II beidseits oder Grad III bis IV unilateral [4].

Abbildung 1. Modifizierte New York Kriterien von 1984 zur Klassifikation der AS [4].

\section{Modifizierte New York Kriterien für ankylosierende Spondylitis (1984)}

\section{Klinische Kriterien:}

1. tiefsitzender Rückenschmerz und Steifigkeit für mehr als 3 Monate mit Besserung durch Bewegung, aber nicht durch Ruhe.

2. Bewegungseinschränkung der LWS in sagittaler und frontaler Ebene.

3. Einschränkung der Thoraxexkursion (alters- und geschlechts-abhängig).

\section{Radiologisches Kriterium:}

Sakroiliitis mindestens Grad 2 beidseits oder Grad 3-4 einseits.

Definitive ankylosierende Spondylitis liegt vor, wenn das radiologische Kriterium und 1 klinisches Kriterium erfüllt sind. 
Zu Beginn des neuen Jahrtausends fehlten evaluierte spezifische Messinstrumente zur Erfassung der Krankheitsaktivität bei AS in klinischen Studien. Die Basis für die heutige Durchführung klinischer Studien im Bereich der AS wurde erst möglich, nachdem eine internationale Gruppe, der ASessemts in SpondyloArthritis international Society (ASAS), die sich umfassend mit den Spondyloarthritiden beschäftigt, Haupt-Outcome-Parameter für Therapiestudien bei AS festgelegt hatte $[5,6]$. Hier inbegriffen sind die verschiedenen Domänen der Erkrankung, die Ziel therapeutischer Intervention sind, wie körperliche Funktionsfähigkeit (BASFI), Schmerz (NRS/ VAS), Krankheitsaktivität (BASDAI), Wirbelsäulenbeweglichkeit (BASMI), Wirbelsäulensteifigkeit/ Entzündung und Müdigkeit. Zusätzlich ist die Verbesserung der Lebensqualität, als Teil der World Health Organisation (WHO)-Gesundheitsdefinition, ein wichtiger Faktor zur Bewertung von Therapieerfolgen bei chronischen Erkrankungen. Der international zurzeit am häufigsten eingesetzte Fragebogen ist der 'Short-Form-36' (SF-36) zur Beurteilung der gesundheitsbezogenen Lebensqualität [7]. Der SF-36-Fragebogen umfasst 9 Dimensionen der Gesundheit: körperliche Funktionsfähigkeit, Rollenverhalten wegen körperlicher Funktionsbeeinträchtigung, Schmerzen, allgemeiner Gesundheitszustand, Vitalität und körperliche Energie, soziale Funktionsfähigkeit, Rollenverhalten wegen seelischer Funktionsbeeinträchtigung, seelische (psychische) Funktionsfähigkeit und Änderung des Gesundheitszustandes. Acht dieser Dimensionen lassen sich in die 2 Hauptdimensionen körperliche Gesundheit (körperliche Funktionsfähigkeit, Rollenverhalten wegen körperlicher Funktionsbeeinträchtigung, Schmerzen, allgemeiner Gesundheitszustand) und mentale/psychische Gesundheit (Vitalität und körperliche Energie, soziale Funktionsfähigkeit, Rollenverhalten wegen seelischer Funktionsbeeinträchtigung, seelische (psychische) Funktionsfähigkeit) zusammenfassen. Ein kürzlich neu entwickelter Score, der Ankylosing Spondylitis 
Disease Activity Score (ASDAS) zur Erfassung der Krankheitsaktivität bei SpA umfasst neben Rückenschmerz im Rahmen der AS, allgemeinem Patientenurteil, peripheren Gelenkschmerzen und Dauer der Morgensteifigeit auch das C-reaktive Protein (CRP) und enthält somit nicht nur subjektive, vom Patienten anzugebende Parameter sondern auch ein objektives Kriterium [8]. Er erfasst noch sensitiver Krankheitsaktivität und Änderungen unter Therapie.

\subsubsection{Therapie der ankylosierenden Spondylitis}

Anfang der 2000er Jahre waren die therapeutischen Optionen der AS nicht weit entwickelt und nicht gut untersucht. Sie bestanden hauptsächlich aus der Gabe nichtsteroidaler Antirheumatika (NSAR) und Krankengymnastik [9]. Nachdem nachgewiesen worden war, dass der zentrale Entzündungsbotenstoff TNF-alpha in entzündeten SIG bei Patienten mit AS stark vermehrt ist [10], wurde in einer offenen „intention-to-treat“ Studie bei 10 Patienten mit aktiver NSAR-refraktärer AS der TNFalpha Blocker Infliximab untersucht [11]. Dies hatte eine rasche und sehr gute Wirksamkeit gezeigt - der BASDAI war insgesamt im 70\% verbessert - und war Ausgangspunkt für eine multizentrische Placebo- kontrollierte Studie mit Infliximab, die diese gute Wirksamkeit bestätigte [12], was auch zur Zulassung des Medikaments in dieser Indikation führte.

Im Gegensatz zu anderen entzündlich-rheumatischen Erkrankungen (zum Beispiel bei der rheumatoiden Arthritis, RA), bei der krankheitsmodifizierende Antirheumatika wie Methotrexat (MTX) oder Sulfasalazin schon gut untersucht waren, existierte keine Vorstellung, ob diese eine Rolle in der Therapie der AS spielen [13, 14]. Die vorhandenen Studien waren teils an kleinen Patientenkollektiven oder mit zu niedriger Dosierung und mit unterschiedlichen, nicht validierten Outcomeparametern durchgeführt 
worden und hatten inkonklusive Ergebnisse erbracht. Am besten untersucht ist Sulfasalazin, welches einen günstigen Effekt auf die peripheren Manifestationen [15] aber nicht auf axialen Manifestationen zeigt [16]. Der Effekt von Sulfasalazin in der Therapie der AS wurde in einer Metaanalyse von 2005 zusammengestellt [14]. In dieser Analyse war in den gepoolten Studien kein signifikanter Effekt für die axialen Manifestationen sichtbar. Ein günstiger Effekt wurde für die periphere Arthritis gefunden. In einer Studie mit Sulfasalazin 2g/Tag versus Placebo bei Patienten, die die European Spondyloarthritis Study Group (ESSG)-Kriterien erfüllten und weniger als 5 Jahre Krankheitsdauer aufwiesen, konnte nochmals gezeigt werden, dass sich keine signifikante Änderung des BASDAI nach 24 Wochen Therapie gegenüber Placebo ergibt [16].

In einer 2004 erstellten Metaanalyse für MTX [13], die 2006 [17] und 2013 [18] ein Update erfahren hat, wurden insgesamt drei randomisierte kontrollierte Studien (RCT) mit Dosierungen zwischen 7.5 und 10mg/ Woche versus Placebo bei insgesamt 116 Patienten und einer Therapiedauer zwischen 12 und 24 Wochen analysiert, hier war kein signifikanter Effekt auf die WS-Beteiligung nachweisbar [19-21]. In einer dieser Studien gab es eine separate Outcome- Messung für periphere Manifestationen, die ebenfalls keine signifikante Besserung zeigte [21]. Ähnlich zeigten sich auch Ergebnisse einer offenen Studie mit MTX bei 34 Patienten und einer Dosis von 12,5 mg/ Woche intramuskulär [22]. Insgesamt sind die Dosierungen, in denen MTX verabreicht wurden, niedrig im Vergleich zu den derzeit verabreichten Dosierungen bei RA, bei der bis 25 mg/ Woche gegeben wird, so dass argumentiert werden kann, dass eine zu niedrige Dosierung des Medikaments in den Studien zur Ineffektivität geführt haben kann.

Systemische Glucocorticoide, die ebenfalls bei anderen entzündlich- rheumatischen Erkrankungen wie RA, systemischer Lupus erythematodes oder bei Vaskulitiden einen 
festen Stellenwert haben, sind nur als intraartikuläre Therapie in entzündete SIG [23] oder in unkontrollierten kleineren Studien in Form von hochdosiertem Methylprednisolon [24-27] untersucht worden. Studien zur Wirksamkeit von oralem Prednisolon existierten nicht [28].

Eine mögliche andere therapeutische Option stellen Bisphosphonate dar. Sie inhibieren nicht nur Osteoklasten, stabilisieren die Knochendichte und beugen osteoporotischen Frakturen vor [29], sondern unterdrücken ebenfalls proinflammatorische Zytokine wie Interleukin (IL)-1, TNF-alpha und IL6 [30]. Sie zeigten antientzündliche Effekte bei RA [29]. Von einer Gruppe aus Kanada waren 2 kleinere Studien und eine kontrollierte Therapiestudie mit dem i.v.- Bisphposphonat Pamidronat, mit positivem Effekt bei Patienten mit AS und SpA berichtet worden [31-33], jedoch gab es bis dato keine Untersuchungen dieser Therapie durch weitere Gruppen.

In der letzten Dekade wurde mit der Entdeckung, dass TNF-alpha-Blocker eine sehr gute Wirksamkeit bei der AS haben, die Grundlage für eine effektive entzündungshemmende Therapie geschaffen [34-36]. 40-50\% der Patienten zeigen eine Antwort gemessen mit dem Summenscore ASAS40 auf eine Therapie mit TNFalpha Blockern $[20,21,24,25]$, dagegen findet sich bei den anderen Patienten kein oder nur ein mäßig gutes Ansprechen primär oder im Therapieverlauf. Daher ist es nötig, für diese Patienten andere medikamentöse Therapien zu finden. In Zusammenarbeit mit der European League against Rheumatism (EULAR), entsprechend der Evidenz im Jahr 2006 hat ASAS Empfehlungen zur Behandlung der Spondyloarthritiden publiziert (Abbildung 4) [37]. Weiterhin wurden von der Gruppe Empfehlungen für den Einsatz von TNF-alpha-Blockern formuliert, im Jahr 2009 aktualisiert und 2011 publiziert (Abbildung 5, 6) [28]. Gemäß diesen Empfehlungen kann nach Versagen zweier NSAR in maximaler Dosierung oder maximaler Verträglichkeit 
über insgesamt vier Wochen die Therapie mit einem TNF-alpha- Blocker begonnen werden. 
Abbildung 5: Empfehlungen der Assessment of SpondyloArthritis international Society (ASAS) / European League Against Rheumatism (EULAR). [37]

\section{ASAS/EULAR Empfehlungen für die Behandlung der ankylosierenden Spondylitis}
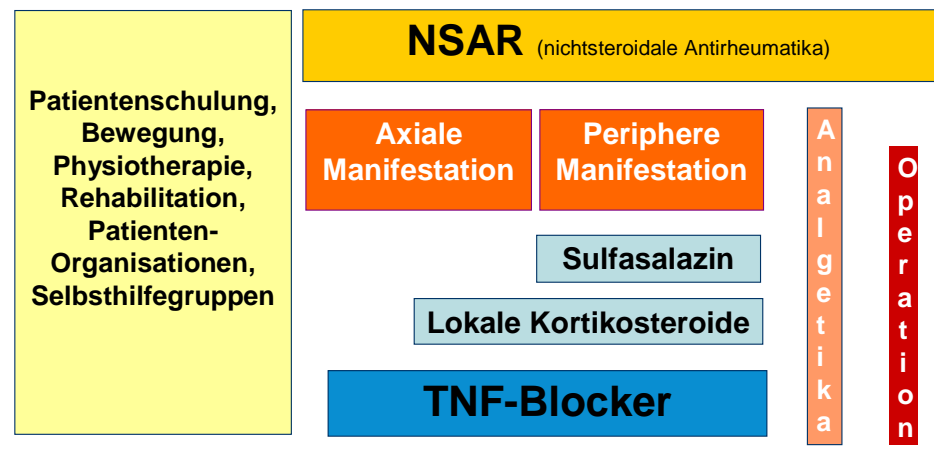

Zochling J et al. Ann Rheum Dis 2006;65:442-52 (mit Genehmigung)

Abbildung 6: ASAS Empfehlungen für die Behandlung von Patienten mit axialer Spondyloarthritis mit TNF alpha Blockern [28].

\section{ASAS-Empfehlung für die Behandlung von AS Patienten mit TNF $\alpha$-Blockern}

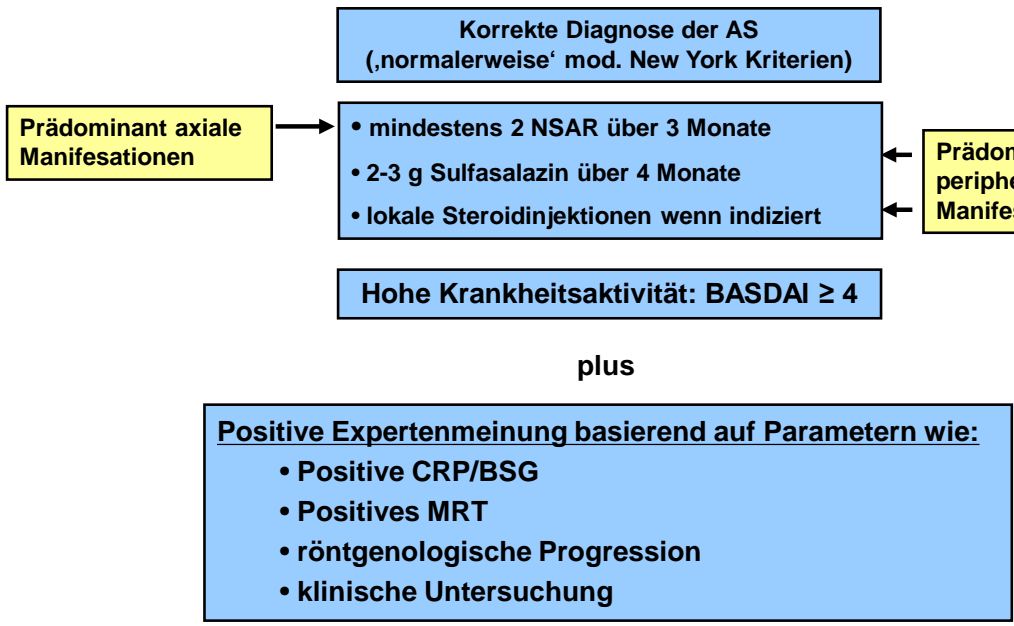




\subsection{Nichtröntgenologische axiale Spondyloarthritis}

\subsubsection{Diagnose der nichtröntgenologischen Spondyloarthritis}

Mit Hilfe der modifizierten New York Kriterien von 1984 können aber nur Patienten klassifiziert werden, bei denen die Entzündung bereits im Röntgenbild sichtbare strukturelle Schädigungen in den SIG, definiert als mindestens Grad II beidseits oder Grad III unilateral, hinterlassen haben, was nicht bei allen Patienten der Fall ist und sich in der Regel erst über mehrere Jahre entwickelt. Da es sich jedoch um ein Krankheitskontinuum handelt, bei dem zunächst eine Entzündung der SIG im Vordergrund steht und erst im Verlauf und nicht bei jedem Patienten knöcherne, im Röntgenbild sichtbare, Veränderungen entstehen, wird die Gesamtgruppe der Erkrankung mit diesen Kriterien nicht erfasst [38], Abbildung 2.

Zudem ist es aus klinischer und therapeutischer Sicht sinnvoll, die Erkrankung in ein vorwiegend axiales und ein vorwiegend peripheres Erkrankungsmuster zu unterteilen, je nachdem ob die Wirbelsäule und SIG oder eine periphere Arthritis, meist Oligoarthritis mit vorwiegendem Befall der unteren Extremität, Enthesitis und/ oder Daktylitis mit weiteren typischen Merkmalen für SpA im Vordergrund stehen. 
Abbildung 2: Konzept der axialen Spondyloarthritis als Krankheitskontinuum [38].

\section{Axiale Spondyloarthritis}

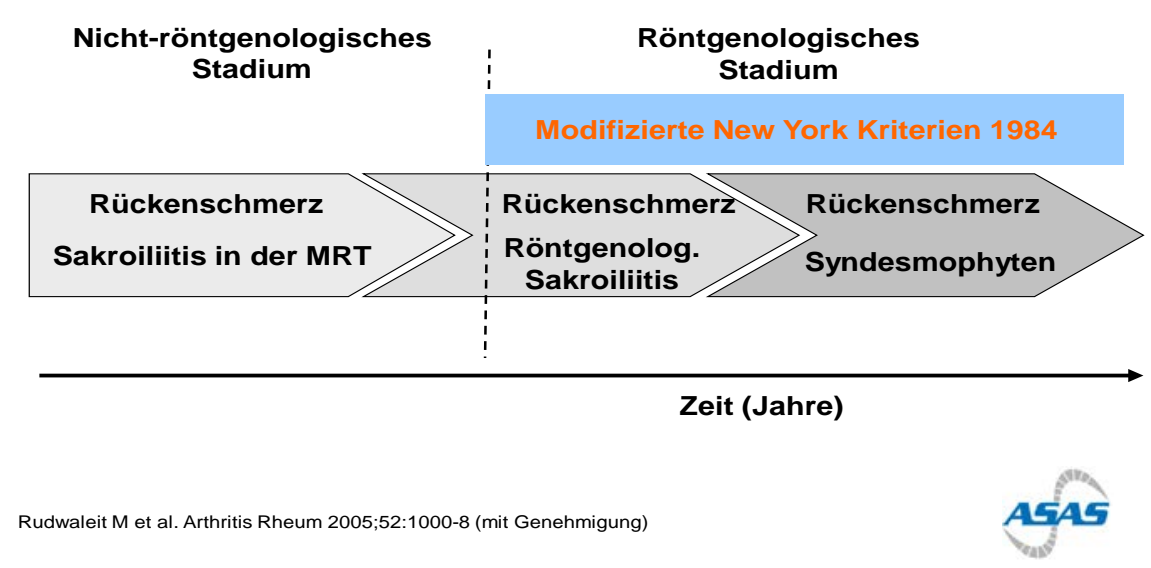

Um diese Gruppe von Patienten mit axialer SpA umfassender, auch ohne Zeichen der Sakroiliitis im Röntgenbild, zu erfassen, wurden neue Klassifikationskriterien für die SpA durch ASAS entwickelt und im Jahr 2009 publiziert. Sie gelten für ein prädominant axiales Befallsmuster [39]. Eingangskriterium für die Klassifikation einer axialen SpA ist ein junges Lebensalter bei Beginn der Symptomatik < 45 Jahre und ein chronischer Rückenschmerz > 3 Monate. Entweder soll eine Sakroiliitis im Röntgenbild (mindestens Grad II bilateral oder Grad III unilateral) oder in der Magnet-Resonanz-Tomographie (MRT) der SIG mit Nachweis aktiver entzündlicher Veränderungen oder ein positives HLA-B27 vorhanden sein. Weitere Parameter sind chronischer entzündlicher Rückenschmerz, der unter anderem durch einen schleichenden Beginn, nächtliche Rückenschmerzen, eine Besserung bei Bewegung, aber nicht in Ruhe sowie alternierende Gesäßschmerzen charakterisiert ist [40]. Andere Parameter umfassen Uveitis, periphere Arthritis, Enthesitis (vorwiegend der Ferse), Daktylitis, Psoriasis, chronisch-entzündliche Darmerkrankung (Morbus Crohn/ Colitis ulcerosa), gutes Ansprechen auf NSAR, positive Familienanamnese für SpA, HLA-B27 und ein erhöhtes 
CRP. Mit diesen Klassifikationskriterien lassen sich sowohl Patienten mit AS gemäß den modifizierten New York Kriterien als auch Patienten im nicht-röntgenologischen Stadium (nr-axSpA) klassifizieren (Abbildung 3).

Abbildung 3: ASAS Klassifikationskriterien der axialen Spondyloarthritis [41].

\section{ASAS Klassifikationskriterien für axiale Spondyloarthritis (SpA)}

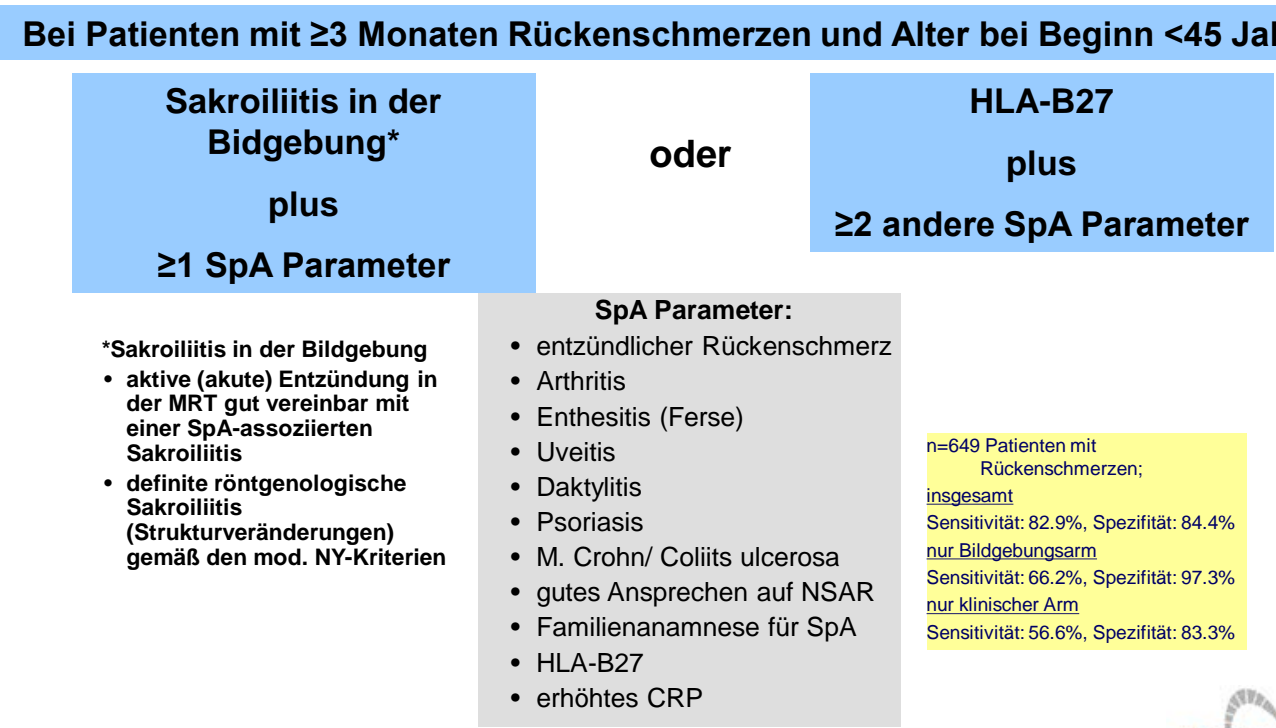

ASAE

Rudwaleit M et al. Ann Rheum Dis 2009;68:777-783 (Mit Genehmigung)

\subsubsection{Therapie der nichtröntgenologischen axialen Spondyloarthritis}

Zirka 40-50\% der Patienten mit aktiver AS sprechen gut auf eine Therapie mit TNFalpha Blockern (z.B. gemessen anhand einer 50\% Verbesserung des BASDAI) an. Im Parallelschluss zur RA wurde die Frage gestellt, ob Patienten mit AS im frühen Krankheitsstadium eine höhere Wahrscheinlichkeit haben, gut auf eine Therapie mit TNF-alpha Blockern anzusprechen.

In den Studien bei Patienten mit AS wurden als Prädiktoren für ein gutes Ansprechen neben einem erhöhten CRP und einem hohen Ausmaß an Entzündung in der MRT, eine 
kurze Krankheitsdauer und junges Lebensalter, welches mit einer kurzen Krankheitsdauer korreliert, identifiziert. Eine 50\% Verbesserung des BASDAI fand sich bei $73 \%$ der Patienten mit einer Krankheitsdauer von unter 10 Jahren im Vergleich zu 31\% der Patienten mit einer Krankheitsdauer über 10 Jahre [42].

Es stellte sich daher die Frage, ob Patienten mit nicht-röntgenologischer axialer SpA, also im Gegensatz zu Patienten mit AS ohne deutliche Zeichen von Sakroiliitis im Röntgenbild, besser auf eine Therapie mit TNF-alpha Blockern ansprechen. Hierzu gab es bislang noch keine Therapiestudien.

Mit eigenen Arbeiten in den 2000-er Jahren zur Therapie der AS und der nr-axSpA mit krankheitsmodifizierenden Antirheumatika, Pamidronat, Prednisolon und dem TNFalpha Blocker Adalimumab habe ich einen relevanten Beitrag zur Verbesserung der therapeutischen Optionen und Einschätzung der therapeutischen Wirksamkeit der Medikamente in der Gruppe der axialen SpA geleistet.

In der hier vorgelegten Schrift sind eigene publizierte Arbeiten in Form einer kumulativen Habilitationsschrift zusammengefasst. Zum besseren Verständnis sind diese Arbeiten in einen Gesamtkontext eingebettet. 


\section{Ergebnisse}

\subsection{Outcome Parameter bei der ankylosierenden Spondylitis}

\subsubsection{Evaluation des Lebensqualitätsfragebogens SF-12 als Outcome Parameter}

bei der ankylosierenden Spondylitis im deutschen Sprachraum

Der Fragebogen zur Messung der Lebensqualität SF36 wurde in einer Placebokontrollierten multizentrischen Studie zur Wirksamkeit des TNF-alpha-Hemmers Infliximab bei 70 Patienten mit AS eingesetzt [12]. 35 Patienten erhielten Placebo, 35 Patienten erhielten Infliximab $5 \mathrm{mg} / \mathrm{kg}$ als Infusion in Woche 0, 2 und 6. Anschließend wurden die Patienten über 12 Wochen offen mit Infliximab $5 \mathrm{mg} / \mathrm{kg}$ i.v. alle 6 Wochen weiterbehandelt. Zur Messung der Krankheitsaktivität bei AS wurde der BASDAI, für die funktionellen Beeinträchtigungen der BASFI, für die Einschränkung der Wirbelsäulenbeweglichkeit der BASMI, für Schmerzen die VAS, das CRP sowie die gesundheitsbezogene Lebensqualität (SF-36) untersucht. Auf der Basis des sehr guten Ansprechens der Patienten auf das Medikament ergaben sich erhebliche Verbesserungen auch in der Patientenbewertung der Lebensqualität.

In einer eigenen Arbeit habe ich analysiert, ob die schneller ausfüllbare kürzere Fragebogenversion SF-12, der im Gegensatz zum SF-36 nur 12 vom Patienten auszufüllende Fragen hat, eine vergleichbare Aussagefähigkeit bezüglich der Summenscores für körperliche und mentale Gesundheit wie die des SF-36 bei Patienten mit AS hat [43]. Dies wäre ein beträchtlicher Vorteil für zukünftige Studien, da Zeit und Belastung für die Patienten beim Ausfüllen des Fragebogens deutlich reduziert wären. Darüber hinaus wurden die Daten der AS-Patienten vergleichbar mit einer Normstichprobe der deutschen Normalbevölkerung.

Aus den gewonnenen Daten des SF-36 wurde der SF-12 extrahiert und mit dem SF-36 bezüglich Korrelation, Validität, Re-Test-Reliabilität und Änderungssensitivität 
verglichen. Die Summendimensionen für körperliche und psychische Gesundheit für SF12 und SF-36 waren gut vergleichbar ( $R=0,96$ bzw. 0,82). Bei Patienten mit AS führte Infliximab zu einer deutlichen Verbesserung aller Outcome-Parameter für Krankheitsaktivität. Alle Einzelkomponenten der gesundheitsbezogenen Lebensqualität verbesserten sich deutlich. Die Daten zeigen, dass bei Studien mit AS-Patienten der SF-12 zur Erfassung der Lebensqualität vergleichbar gut einsetzbar ist.

\section{Eigene Referenz:}

Haibel H, Niewerth M, Brandt J, Rudwaleit M, Listing J, Sieper J, Braun J., Measurement of quality of life in patients with active ankylosing spondylitis being treated with infliximab- a comparison of SF-36 and SF-12 Z Rheumatol. 2004 Oct;63(5):393401. German.

http://dx.doi.org/10.1007/s00393-004-0604-5 


\subsection{Alternative Therapieansätze bei der ankylosierenden Spondylitis}

\subsubsection{Pamidronat bei ankylosierender Spondylitis als alternativer Therapieansatz}

Dem Bisphosphonat Pamidronat werden antientzündliche Effekte zugeschrieben [29]. In 3 kanadischen monozentrischen Studien fanden sich Hinweise auf eine Effektivität des Medikaments bei SpA/ AS [31-33]. In einer eigenen Studie behandelten wir 12 Patienten mit fortgeschrittener AS und erhöhter Krankheitsaktivität, gemessen mittels BASDAI $\geq 4$ und einer mittleren Krankheitsdauer von 20 Jahren (2-41), mit Pamidronat 60 mg an den Tagen 0, 2, 14, 28 und 56 [32]. Die Gesamtbeobachtungsdauer betrug 6 Monate. Bei 5 Patienten erfolgten MRT-Untersuchungen mit Kontrastmittel der WS und SIG, bei allen 5 Patienten fanden sich Zeichen der Aktivität in Form von subchondralen Knochenmarködemen in der Short Tau Inversion Recovery (STIR)- bzw. postGadolinium-Kontrastmittel Sequenz. Zudem wurden Dual energy X-ray Absorptiometry (DXA)- Knochendichtemessungen der Hüfte und der WS bei allen Patienten vor Studienbeginn durchgeführt. Drei Patienten hatten demnach eine Osteoporose und 5 Patienten eine Osteopenie.

Signifikante Besserungen von Aktivitätsindizes wurden lediglich nach 3 jedoch nicht nach 6 Monaten gefunden. Der mittlere BASDAI änderte sich von 5.5 auf $4.6(p=0.04)$ nach 3 Monaten und auf 4.7, p=ns nach 6 Monaten. Ebenfalls nach 3 Monaten änderte sich signifikant das allgemeine Arzturteil $(\mathrm{p}=0.035)$ und der Lebensqualitätsfragebogen SF-12 für körperliche Gesundheit ( $p=0.047)$. Insgesamt war eine geringe Besserung der Krankheitsaktivität nur nach 3 jedoch nicht mehr nach 6 Monaten zu erfassen. Bei den meisten Patienten blieb der BASDAl über 4. Dies bedeutet eine erhöhte Krankheitsaktivität, wie sie die Voraussetzung für eine eventuelle Therapie mit TNFalpha-Blockern ist. 
Im Verlauf wurden noch 4 weitere offene Studien publiziert. In der ersten dieser Studien wurden 15 Patienten mit aktiver AS mit 60 mg Pamidronat über 6 Monate behandelt [44]. Der mittlere BASDAI verbesserte sich signifikant jedoch nicht klinisch relevant von 6.8 auf 5.8, andere Outcomeparameter verbesserten sich nicht. In der zweiten Studie wurden insgesamt 35 Patienten, davon 26 mit AS und 9 mit nicht-röntgenologischer Form (erfüllten die European Spondyloarthritis Study Group (ESSG)-Kriterien [45]) offen mit Pamidronat 60 mg über 6 Monate behandelt. In dieser Studie verbesserte sich der BASDAI bereits nach 3 Monaten signifikant, eine 50\% Verbesserung des BASDAI fand sich bei 23\% der Patienten nach 3 Monaten und bei 17\% der Patienten nach 6 Monaten. Das CRP war fluktuierend und wies keine signifikanten Veränderungen auf [46]. In der dritten Studie mit 21 AS Patienten mit ausschließlich axialem Befall wurden ebenfalls mit 60 mg Pamidronat monatlich über 6 Monate behandelt. In dieser Studie erreichten 19\% der Patienten eine 20\% Verbesserung nach den ASAS-Kriterien, keiner erreichte ASAS40 [47]. In einer vierten Studie aus Indien wurden 35 AS-Patienten monatlich mit Pamidronat 60 mg iv. behandelt. Von diesen erreichten 85\% ASAS20, 77\% eine 50\% Verbesserung des BASDAI, die BSG sank bei $54 \%$ und das CRP bei 73\% der Patienten nach 6 Monaten Therapie. Bei Patienten mit peripherer Arthritis besserte sich diese ebenfalls im Verlauf deutlich [31].

Zusammenfassend findet sich ein unterschiedliches Ansprechen in den verschiedenen Studien. In einigen Studien zeigte sich ein Therapieerfolg einzelner Komponenten zumeist erst nach 4 Monaten Behandlungsdauer [23, 33, 48-51], in anderen kein Therapieerfolg [eigene Referenz, [44]. Deutlich wird, dass der Therapieerfolg klar dem der TNF-Blocker nachsteht, bei denen eine deutliche Besserung der Krankheitsaktivität (BASDAI 50, ASAS40) bei ca. 50\% der Patienten erreicht wird [9, 36, 52, 53]. Somit ist Pamidronat auch nicht in die Therapieempfehlungen für AS aufgenommen worden [37]. 


\section{Eigene Referenz:}

Haibel H, Brandt J, Rudwaleit M, Soerensen H, Sieper J, Braun J. Treatment of active ankylosing spondylitis with pamidronate. Rheumatology (Oxford). 2003 Aug;42(8):101820.

http://dx.doi.org/10.1093/rheumatology/keg256 


\subsubsection{Methotrexat bei ankylosierender Spondylitis}

Wie eingangs erwähnt, waren im Gegensatz zu anderen entzündlich rheumatischen Erkrankungen (zum Beispiel RA) bei der AS Disease Modifying Antirheumatic Drugs (DMARDs) nur wenig untersucht $[13,14]$. Bei der Therapie der RA mit DMARDs wird normalerweise nach einer Behandlungsdauer von 3 Monaten eingeschätzt, ob die Therapie wirksam ist oder eine Therapieänderung vorgenommen werden soll [54].

In einer eigenen offenen Studie habe ich daher 20 Patienten mit aktiver NSARrefraktärer AS über 16 Wochen mit MTX behandelt [55]. Zunächst erhielten die Patienten MTX 15 mg subkutan über 1 Monat und anschließend MTX 20 mg subkutan über weitere 3 Monate. Der mittlere BASDAI zu Baseline betrug 5.6 (Spannweite 4-9.3). Der primäre Outcome-Parameter war eine Verbesserung gemessen anhand der ASAS 20 Kriterien nach 4 Monaten.

Dieses Kriterium wurde von 25\% der Patienten erreicht. ASAS 40 wurde von 10\% der Patienten erreicht, ein Ausmaß des Therapieeffekts, welches auch bei PlaceboTherapie in anderen Studien erreicht wurde, und partielle Remission anhand der ASAS Kriterien wurde von keinem Patienten erreicht. Der mittlere BASDAI blieb im Vergleich zu Baseline unverändert. Für andere klinische Outcomeparameter und das CRP war ebenfalls keine Verbesserung festzustellen. Es fand sich eine geringe, nicht signifikante Reduktion der Anzahl geschwollener Gelenke. Insgesamt konnte kein Effekt für die axialen Manifestationen und Entzündungsparameter bei Patienten mit aktiver AS über das zu erwartende Ausmaß einer Placebo-Antwort hinaus festgestellt werden. Besonders basierend auf den Ergebnissen dieser Studie wird MTX für die Therapie der ax-SpA in den internationalen ASAS/EULAR Empfehlungen als unwirksam eingeschätzt $[28,37]$. 


\section{Eigene Publikation:}

Haibel H, Brandt HC, Song IH, Brandt A, Listing J, Rudwaleit M, Sieper J. No efficacy of subcutaneous methotrexate in active ankylosing spondylitis - a 16 weeks open label trial. Ann Rheum Dis. 2007 Mar;66(3):419-21.

http://dx.doi.org/10.1136/ard.2006.054098 


\subsubsection{Steroide bei der Therapie der ankylosierenden Spondylitis am Beispiel von Prednisolon}

Während es einige Studien mit intravenösem hochdosiertem Methylprednisolon gibt, die einen guten und anhaltenden Effekt bei Patienten mit aktiver AS zeigen [50-53], sowie ebenfalls Studien zur guten Effektivität lokal CT-gesteuerter Injektionen von Steroiden ins entzündete Sakroiliakalgelenk [35], waren bis kürzlich keinerlei Studien zu oral verabreichten moderaten Dosierungen von Prednisolon (20-50mg/Tag) bei Patienten mit AS verfügbar [22].

In einer eigenen Placebo-kontrollierten, randomisierten doppelblinden Studie bei Patienten mit aktiver AS wurden 36 Patienten entweder mit Placebo ( $n=14)$, Prednisolon 20 mg p.o. ( $n=13)$ oder Prednisolon $50 \mathrm{mg}$ p.o. $(n=12)$ über insgesamt 2 Wochen behandelt [56].

Unter Zuhilfenahme eines „Multiple Endpunkt“- Tests, der Krankheitsaktivitäts-, Beweglichkeits-, Funktions- sowie Entzündungsparameter zusammenfasste (BASDAI, ASDAS, BASFI, Schmerz, allgemeines Patientenurteil, Morgensteifigkeit, BASMI und CRP) konnte eine signifikante Besserung $(p=0.017)$ in der Prednisolon 50 mg- Gruppe im Vergleich zu Placebo nachgewiesen werden. Der Unterschied in der 20 mg Gruppe im Vergleich zu Placebo erreichte keine statistische Signifikanz $(p=0.20)$. In beiden Prednisolon Gruppen sank das CRP signifikant, dies erklärt sicher auch die signifikante Verbesserung des ASDAS, da dieser das CRP als Parameter enthält. Zusammenfassend kann gesagt werden, dass eine relativ hohe Dosis Prednisolon (50 mg) eine gewisse Effektivität bei Patienten mit aktiver AS, insbesondere bei den CRPpositiven Patienten erbracht hat, während dies in der 20 mg Gruppe nicht beobachtet wurde. Ob der klinische Effekt nach 50 mg Prednisolon per os (p.o.) über 2 Wochen hinaus anhält, oder ob eine niedrige Erhaltungsdosis unterhalb der Cushingschwelle zu 
anhaltender Verbesserung führt, wurde in dieser Studie nicht untersucht. Eine längerfristige Therapie mit Prednisolon 50 mg p.o. wäre aber wegen des erhöhten Nebenwirkungsrisikos nicht akzeptabel.

Eigene Referenz: Haibel H, Fendler C, Listing J, Callhoff J, Kraus A, Braun J, Sieper J. Efficacy of oral prednisolone in active ankylosing spondylitis - Results of a double blind placebo controlled short term trial. Ann Rheum Dis. 2013 May 16.

http://dx.doi.org/10.1136/annrheumdis-2012-203055 


\subsection{TNF-alpha Blocker in der Therapie der ankylosierenden Spondylitis}

\subsubsection{Effektivität von Infliximab in der Routineversorgung bei Patienten mit}

aktiver ankylosierender Spondylitis

Mit der Entdeckung, dass TNF-alpha-Blocker eine sehr gute Wirksamkeit bei der AS haben, wurde die Grundlage für eine effektive entzündungshemmende Therapie geschaffen [34-36]. Zusätzlich zur Kurzzeiteffektivität wurde im Rahmen der Studien die Langzeiteffektivität und -Sicherheit über bis zu 7 Jahre bei verschiedenen TNF-alpha Blockern publiziert [57-59].

Nachdem in den Placebo- kontrollierten Zulassungsstudien bei Patienten mit engen Einund Ausschlusskriterien eine gute Wirksamkeit von TNF-Blockern nachgewiesen wurde, ging es nun darum, die Effektivität in nicht auf SpA spezialisierten rheumatologischen Zentren unter täglichen Praxisbedingungen zu überprüfen.

In einer eigenen offenen multizentrischen Studie wurde der TNF-alpha Blocker Infliximab bei Patienten mit aktiver AS über 24 Wochen untersucht [60]. Primärer Outcomeparameter war die Verbesserung der Lebensqualität gemessen mit dem SF36. Zudem wurden die täglichen Aktivitäten und die Arbeitsproduktivität mittels „Work Productivity and Activity Index" (WPAI) sowie BASDAI, BASFI, BASMI, allgemeines Patientenurteil, Verbesserungen des ASAS Score, Schmerz und Entzündungsparameter (CRP) untersucht.

Hierfür wurden 101 Patienten mit aktiver Erkrankung (mittlerer BASDAI 6.3, Spannweite 4.0 bis 9.8) eingeschlossen. Infliximab $5 \mathrm{mg} / \mathrm{kg}$ Körpergewicht (KG) wurde in den Wochen 0, 2, 6, 12, 18 und 24 intravenös verabreicht und der Therapieeffekt wurde in Woche 28 ausgewertet. 
Der SF-36 Score für körperliche Gesundheit besserte sich von $27.6 \mathrm{zu}$ Baseline auf $40.9(p<0.001)$ und der mittlere Score für mentale Gesundheit von 44.4 auf 53.0 $(p<0.001)$. ASAS 40 wurde von $60.4 \%$ und die ASAS-Kriterien für partielle Remission wurden von $27.7 \%$ der Patienten erreicht. Eine Besserung um 50\% des initialen BASDAI erreichten $66.6 \%$ der Patienten. Ähnliche deutliche Verbesserungen fanden sich des Weiteren für den mittleren BASDAI, BASFI, BASMI, allgemeines Patientenund Arzturteil, CRP und WPAI. 11.8\% der Patienten beendeten die Studie vorzeitig wegen unerwünschten Ereignissen.

Insgesamt zeigte die Therapie mit Infliximab eine hohe Effektivität auch in nichtspezialisierten Zentren und in täglicher Praxis während eines Behandlungszeitraums von 24 Wochen. Die Lebensqualität verbesserte sich für die große Mehrheit der Patienten erheblich. Dies war sehr ähnlich im Vergleich zu der Placebo- kontrollierten Studie mit Infliximab [61], in der ebenfalls signifikante Verbesserungen für körperliche und psychische Gesundheit nach 24 Wochen eingetreten waren. Im Vergleich zu den Placebo- kontrollierten Zulassungsstudien, in denen eine $50 \%$ Verbesserung des BASDAI bei $52.9 \%$ der Patienten [12] beziehungsweise bei $51 \%$ der Patienten [62] nach einem halben Jahr Behandlung erreicht wurde, erreichten nach 28 Wochen diese Verbesserung in der vorliegenden Studie mehr Patienten (66.3\%). Diese deutlichere Verbesserung ist wiederum vergleichbar zu anderen offenen Studien mit Infliximab, in denen z.B. eine ASAS50 Verbesserung nach 24 Wochen von bis zu $86 \%$ der Patienten erreicht wurde. Allerdings hatten bei Studienbeginn in dieser Studie alle Patienten ein erhöhtes CRP [63].

\section{Eigene Publikation:}


Haibel H, Song IH, Rudwaleit M, Listing J, Hildeman S, Sieper J, Multicenter open label study with infliximab in active ankylosing spondylitis over 28 weeks in daily practice. Clin Exp Rheumatol. 2008 Mar-Apr;26(2):247-52.

http://www.clinexprheumatol.org/article.asp?a=3311 


\title{
Multicenter open-label study with infliximab in active ankylosing spondylitis over 28 weeks in daily practice
}

\author{
H. Haibel ${ }^{1}$, I.H. Song ${ }^{1}$, M. Rudwaleit ${ }^{1}$, J. Listing ${ }^{2}$, S. Hildemann ${ }^{3}$, J. Sieper ${ }^{1}$
}

${ }^{\text {I} M e d i c a l ~ D e p a r t m e n t ~ I, ~ R h e u m a t o l o g y, ~ C h a r i t e ́, ~ C a m p u s ~ B e n j a m i n ~ F r a n k l i n ~ H o s p i t a l, ~ B e r l i n ; ~}$ ${ }^{2}$ German Rheumatism Research Centre, Berlin; ${ }^{3}$ Essex Pharma, München, Germany.

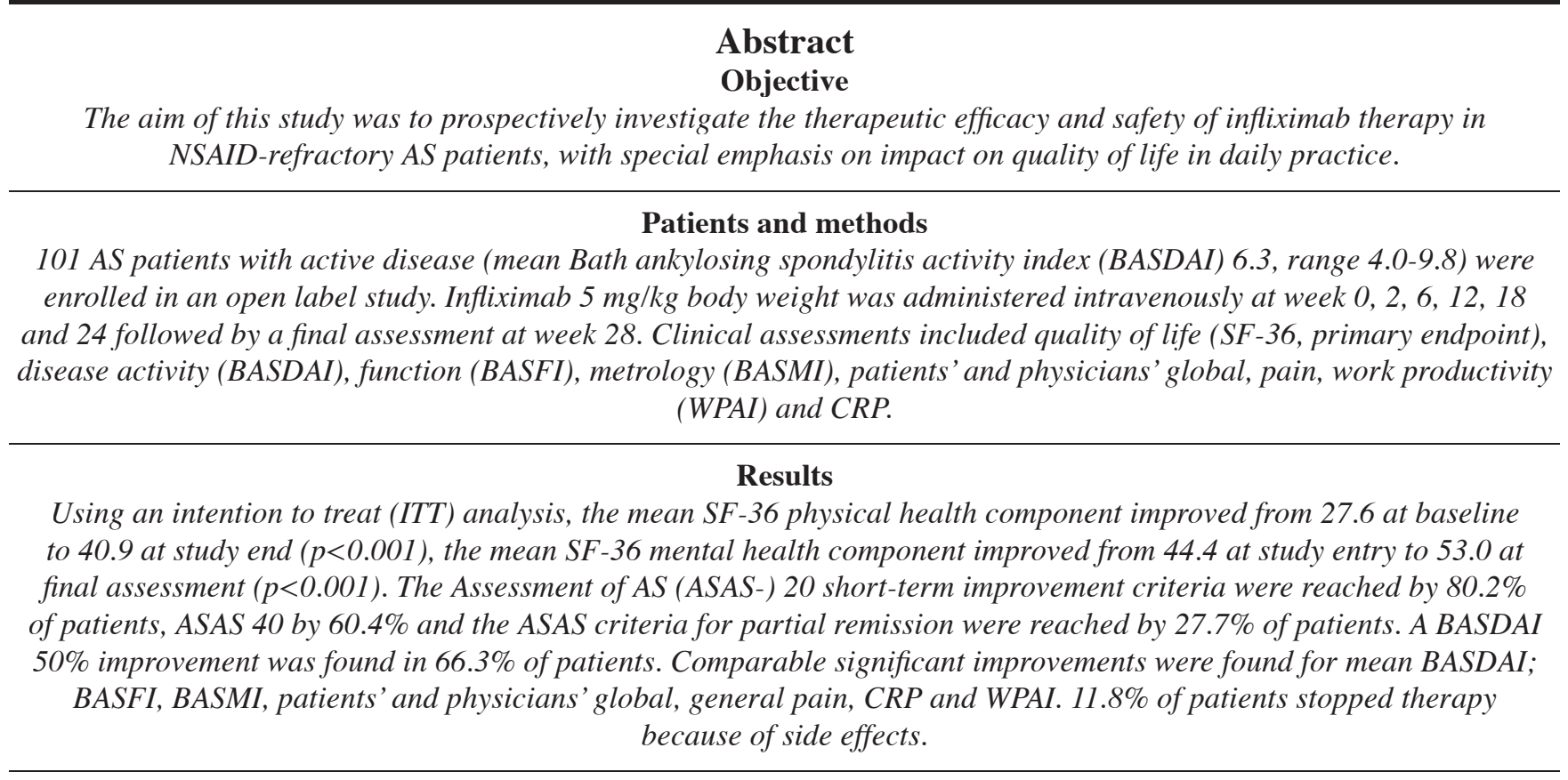

\section{Conclusions}

Infliximab showed high efficacy and safety when used by non-specialised rheumatologists in daily practice.

\section{Key words}

Infliximab, ankylosing spondylitis, open-label trial, daily practice. 
Hildrun Haibel, MD; In-Ho Song, MD; Martin Rudwaleit, MD; Joachim Listing, PhD; Steven Hildemann, MD;

Joachim Sieper, Professor.

This study was supported by Essex Pharma.

Please address correspondence and reprint requests to: Hildrun Haibel, MD, Medical Department I, Rheumatology, Charité, Campus Benjamin Franklin, Hindenburgdamm 30, 12200 Berlin, Germany.

E-mail:hildrun.haibel@charite.de

Received on March 28, 2007; accepted in revised form on September 19, 2007.

(C) Copyright CLINICAL AND

EXPERIMENTAL RHEUMATOLOGY 2008.

Conflict of interest:

Dr. H. Haibel has received honoraria for presentations at scientific symposia and financial support for scientific studies from Schering-Plough and Abbott;

Dr. M. Rudwaleit has received honoraria for presentations at scientific symposia, participation in advisory board meetings, and financial support for scientific studies; Dr. S. Hildemann is employed by Essex-Pharma, the distributor of infliximab in Europe, but has no involvement in the marketing and sales of products;

Dr. J. Sieper has received honoraria for consultancies and financial support for scientific studies from Schering-Plough, Wyeth, Abbott, Roche and BMS; Drs. I.H. Song and J. Listing have declared no competing interests.

\section{Introduction}

Ankylosing spondylitis (AS) is a chronic inflammatory rheumatic disease, which primarily affects the sacroiliac joints (SIJ) and spine in young adults between 20 and 40 years of age. Similar to rheumatoid arthritis (RA) patients, AS is characterized by pain, functional impairment and fatigue which leads to reduced quality of life, high morbidity $(1,2)$, and loss of work productivity $(3)$. Treatment options are limited in AS (4, 5). Until recently, conventional therapy consisted of symptomatic non-steroidal anti-inflammatory drugs (NSAIDs) and physical therapy. Tumour necrosis factor (TNF)-alpha blocking agents have now been shown to be highly effective and well tolerated in patients with active AS (6-8), Initial studies were conducted with infliximab for this indication (9). The results were confirmed in two placebo controlled studies $(7,10)$ and infliximab has been approved for the treatment of AS in Europe and the US.

The aim of this study was to investigate the influence of therapy with infliximab over 24 weeks on quality of life, daily activities, and work productivity as well as on efficacy and safety when used in daily practice by rheumatologists in private practices.

\section{Methods}

Study design

A 28 week, prospective, multicentre (21 centres) open-label trial started in October 2002. One hundred and one patients (age 18-60 years) with AS according to the modified New York Criteria (11) were included. Inclusion required a Bath Ankylosing Spondylitis Disease Activity index (BASDAI) $(12,14), \geq 4$ and question 2 of the BASDAI for back pain $\geq 4$, despite adequate NSAID therapy. The use of concomitant corticosteroids and disease modifying antirheumatic drugs (DMARDs) were not allowed. Exclusion criteria included evidence of latent tuberculosis (negative chest $\mathrm{X}$-ray during the last 4 weeks before study start and negative 10 IU Mendel Mantoux tuberculine (PPD) skin test), any uncontrolled concomitant disease, pregnancy, breastfeeding or relevant changes according to clinical and laboratory examinations. Local ethical committee approval and informed consent were obtained prior to enrollment in the study. Patients received infliximab $5 \mathrm{mg} / \mathrm{kg}$ body weight at baseline, weeks 2, 6, 12, 18, 24, followed by a follow-up visit at week 28 .

\section{Clinical outcome assessments}

Quality of life (SF-36) was primary endpoint (13). Secondary endpoints were disease activity (BASDAI), Bath Ankylosing Spondylitis Functional Index (BASFI) $(12,14)$, general and back pain on a numeric rating scale (NRS), patients and physicians global assessment (NRS), Bath Ankylosing Spondylitis Metrology Index (BASMI) (12, 14), C-reactive Protein (CRP), a 50\% improvement in BASDAI, a $20 \%$ and $40 \%$ improvement according to the AS-

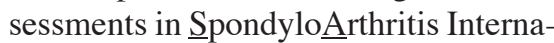
tional Society (ASAS) for spondyloarthritis criteria (15), and ASAS partial remission (value of $<20$ on a $0-100$ scale in each of the four ASAS domains). An exploratory endpoint was the work productivity and activity impairment (WPAI-SHP) questionnaire which is based on a questionnaire addressing the status of employment, hours missed at work during the last week because of AS, and how much the disease affected the productivity at work and daily activities (16). The work productivity impairment score measures how much the amount and type of work is impaired because of $\mathrm{AS}$ on a 0 to 10 numeric rating scale $(0=$ no impairment; $10=$ maximum impairment).

\section{Statistics}

Statistics were performed as an intention-to-treat analysis, last observation carried forward (ITT-LOCF). The nonparametric Wilcoxon signed rank test was used to compare changes between baseline and after-treatment values. A paired $t$-test was used to compare outcomes within different subgroups. Statistical significance was determined as a two-sided $p<0.05$.

\section{Results}

Of 134 patients screened, 101 participated in the treatment phase (for characteristics see Table I) and were analysed 
Table I. Patient characteristics (ITT population).

\begin{tabular}{ll}
\hline Number of patients, $\mathrm{n}$ & 101 \\
Male / female & $79 / 22$ \\
Mean age, years (range) & $49.9(21-67)$ \\
Mean disease duration, years (range) & $15,5(0.6-46)$ \\
BASDAI, mean (range) & $6.3(4.0-9.8)$ \\
HLA-B27 positive (\%) & $87.1 \%$ \\
History of uveitis (\%) & 27.7 \\
Psoriasis (present or previous \%) & 13 \\
IBD (present or previous \%) & 8 \\
CRP > 10 mg/l (\%) & 59,4 \\
Number of patients with history of DMARDs (\%) & 32 \\
Number of patients with previous sulfasalazine (\%) & 18 \\
Number of patients with previous methotrexate (\%) & 7
\end{tabular}

BASDAI: Bath ankylosing spondylitis disease activity index; IBD: inflammatory bowel disease; CRP: C-reactive protein; DMARDs: Disease modifying antirheumatic drugs.

Table II. Primary and Secondary outcome parameters in patients with active ankylosing spondylitis. Mean values.

\begin{tabular}{|c|c|c|c|c|c|}
\hline Outcome parameter & Baseline & Week 2 & Week 12 & Week 24 & Week 28 \\
\hline \multicolumn{6}{|l|}{ SF-36 } \\
\hline Physical sum score & 27.6 & $33.0 * *$ & $39.1 * *$ & $39.2 * *$ & $40.9 * *$ \\
\hline Physical function & 41.4 & $53.5^{* *}$ & $60.5 * *$ & $60.3 * *$ & $64.1 * *$ \\
\hline Physical role function & 21.3 & $28.2 *$ & $64.6 * *$ & $63.1 * *$ & $69.1 * *$ \\
\hline Physical pain & 23.4 & $35.6^{* *}$ & $57.5 * *$ & $60.4 * *$ & $62.9 * *$ \\
\hline Vitality & 31.3 & $39.8 * *$ & $56.4 * *$ & $56.2 * *$ & $58.5 * *$ \\
\hline Mental sum score & 44.4 & $45.3 * *$ & $52.5 * *$ & $53.1 * *$ & $53.0 * *$ \\
\hline Emotional role function & 57.7 & 59.7 & $86.1 * *$ & $87.8 * *$ & $86.5 * *$ \\
\hline Social function & 52.8 & $59.8^{*}$ & $76.0 * *$ & $78.1 * *$ & $78.5 * *$ \\
\hline Mental well being & 58.6 & $61.7 *$ & $73.0 * *$ & $73.3 * *$ & $75.1 * *$ \\
\hline Perception of general health & 37.5 & $47.7 * *$ & $54.5 * *$ & $55.1 * *$ & $55.7 * *$ \\
\hline \multicolumn{6}{|l|}{ WPAI } \\
\hline Employed patients (\%) & 61.4 & 62.4 & 67.0 & 70.0 & NA \\
\hline Missing work hours & 4 & 4 & 2 & 2 & NA \\
\hline Work productivity & 5.2 & $3.7 * *$ & $2,4 * *$ & $2.3^{* *}$ & $2.3 * *$ \\
\hline Daily activities & 6.3 & $4.6^{* *}$ & $3,5 * *$ & $3.3 * *$ & $2.9 * *$ \\
\hline \multicolumn{6}{|l|}{ BASDAI } \\
\hline Summary Score BASDAI & 6.3 & $4.0 * *$ & $3.4 * *$ & $2.9 * *$ & $2.6 * *$ \\
\hline Fatigue (BASDAI Q1) & 6.7 & $4,8 * *$ & $3.6 * *$ & $3.3 * *$ & $3.1 * *$ \\
\hline BASDAI $50 \% * * *$ & NA & $33.7 * * *$ & $60.4 * * *$ & $58.4 * * *$ & $66.3 * * *$ \\
\hline \multicolumn{6}{|l|}{ ASAS criteria } \\
\hline ASAS $20 * * *$ & NA & $57.4 * * *$ & $66,3 * * *$ & $80.2 * * *$ & NA \\
\hline ASAS $40 * * *$ & NA & $38.6 * * *$ & $50,5 * * *$ & $60.4 * * *$ & NA \\
\hline ASAS partial remission $* * *$ & NA & $5,0 * * *$ & $25,7 * * *$ & $27.7 * * *$ & NA \\
\hline BASFI & 5.9 & $4.5 * *$ & $3.7 * *$ & $3.5^{* *}$ & NA \\
\hline BASMI & 4.7 & $4.0 * *$ & $3.4 * *$ & $3.4 * *$ & NA \\
\hline General pain & 7.2 & $4.4 * *$ & $3.5 * *$ & $3.5^{* *}$ & 2.8 \\
\hline Patient's global assessment & 7.6 & $4.5 * *$ & $3.7 * *$ & $3.0 * *$ & NA \\
\hline Physician's global assessment & 6.7 & $3.8 * *$ & $2.6^{* *}$ & $2.4^{* *}$ & NA \\
\hline
\end{tabular}

ASA: Asessment in ankylosing spondyltitis; BASDAI: Bath ankylosing spondylitis disease activity index; BASFI: Bath ankylosing spondylitis functional index; BASMI: Bath ankylosing spondylitis metrology index; SF-36: Short Form-36;

$* p<0.05, v s$. baseline; $* * p<0.001, v s$. baseline; $* * * \%$ of patients; NA: not applicable.

within an ITT-LOCF. Of these 101 patients 12 patients dropped out before the end of the study because of side effects and one patient because he was lost at follow-up.

\section{Primary endpoint}

The SF-36, demonstrated a significant improvement in both the physical and the mental component (physical health: baseline 27.6 vs. 40.9 at week
28, $p<0.001$; mental health: baseline 44.4 vs. 53.0 after 28 weeks, $p<0.001$ ). Likewise all subscales of the SF-36 improved significantly (Table II).

\section{Secondary endpoints}

For the different questions of the WPAI, at baseline $61.4 \%(n=62)$ of the patients had been employed compared to $69.3 \%(n=75)$ at visit $6(p<0.001)$. Of the initially 62 patients who were employed at baseline 4 patients did not work at study end $(6.5 \%)$. The patients who were employed at study entry reported 4 missing work hours due to the disease during the past 7 days at baseline compared to 2 hours at study end $(p=\mathrm{ns})$. There was a clear and statistically significant improvement on work productivity from 5.2 at baseline to $2.3(p<0.001)$ after 28 weeks and on patients' daily activities from 6.3 at baseline to $2.9(p<0.001)$ at study end (Table II).

The mean BASDAI decreased from 6.3 to 2.6 ( $p<0.001)$ for the whole group of patients (Table II). Similarly, after 28 weeks a BASDAI 50\% improvement could be observed in $66.3 \%$ of patients (Table II). Likewise, the ASAS response criteria confirmed the significant improvement at week 24 (80.2\% ASAS $20,60.4 \%$ ASAS 40 and $27.7 \%$ ASAS criteria for partial remission, Table II). For the other secondary outcome parameters see Table II and Figure 1.

Focussing on the subgroup of patients who reached a BASDAI 50 improvement at week $12(69.4 \%$ of patients of the whole group) the mean BASDAI decreased from 6.3 at baseline to 1.6 at week $28(p<0.001)$ compared to the subgroup of patients who did not reach a BASDAI 50 improvement. In the latter group the mean BASDAI decreased only from 6.2 at baseline to 4.0 at week 28 , but still reaching significance $(p<0.001)$ (Fig. 1a). It is of special interest that there was also a significant and considerable decrease of the BASFI from 5.6 to 2.2, $p=0.000$ ) (Fig. 1b) and even of the BASMI (from 4.3 to $2.7 ; p=0.000)$ in the BASDAI responder subgroup. In this responder group the physical component of the SF-36 also improved clearly (from 29 to 46, $p=0.000$ ), while this was not as clear 

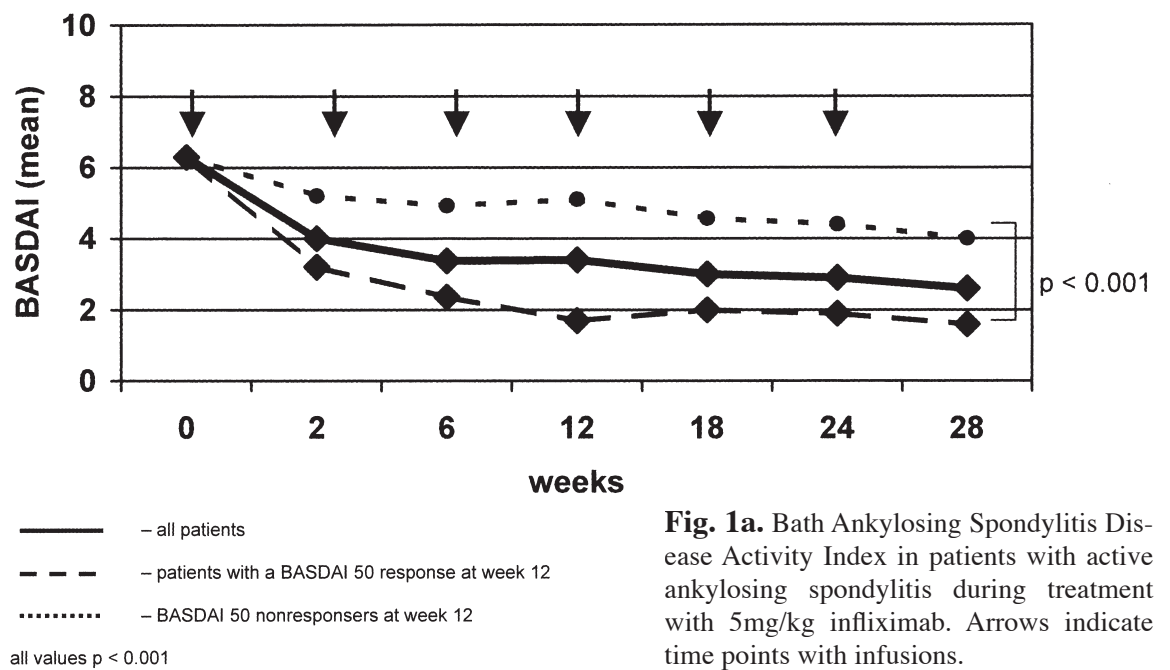

all values $p<0.001$

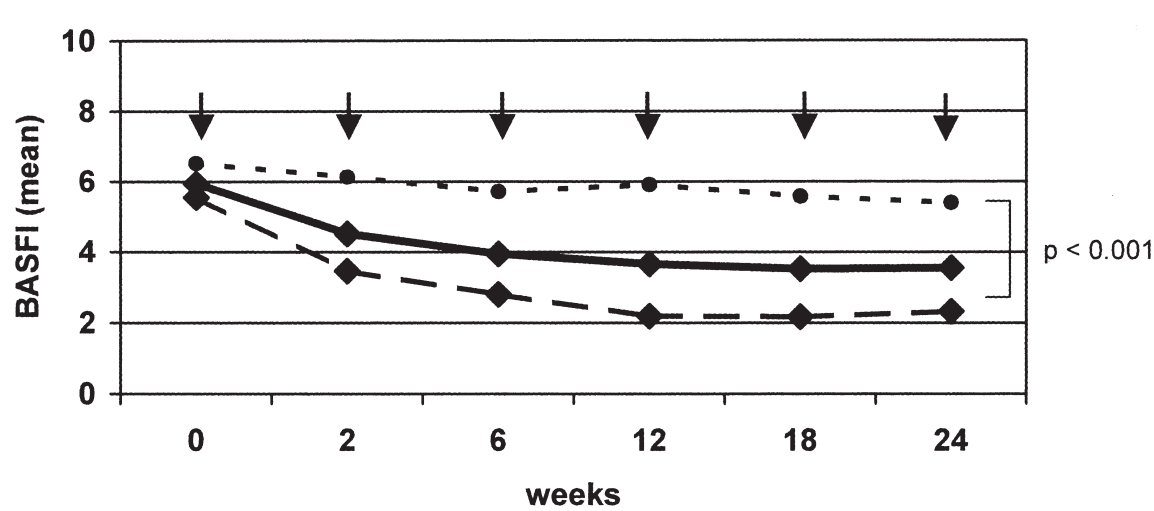

Fig. 1a. Bath Ankylosing Spondylitis Disease Activity Index in patients with active ankylosing spondylitis during treatment with $5 \mathrm{mg} / \mathrm{kg}$ infliximab. Arrows indicate time points with infusions.

Fig. 1b. Bath Ankylosing Spondylitis Functional Index in patients with active ankylosing spondylitis during treatment with $5 \mathrm{mg} / \mathrm{kg}$ infliximab. Arrows indicate time points with infusions.

the mental part of the SF-36 (from 44 to $55, p=0.000)$. Similar differences could be observed when looked in the ASAS responder group (data not shown).

Sixty-six patients $(81 \%)$ who reached BASDAI 50 at week 6 also reached BASDAI 50 at study end and of the patients who did not reach a $20 \%$ improvement of their initial BASDAI at week 6 only one patient $(5 \%)$ reached BASDAI 50 at study end.

When comparing the subgroup of patients who were employed at baseline with the subgroup of patients who were not employed, both had a similar BASDAI at baseline (6.00 vs. 6.64, $p=0.02$ ), but the BASFI was significant lower in the employed group (4.2 vs. $6.4, p<0.001)$. At study end patients who were employed at baseline had a significant lower BASDAI (2.1 vs. 3.4, respectively, $p<0.001)$ and BASFI (2.7 withdrawal from the study in 5 cases: allergic infusion related reactions in 2 , pneumonia in 2 and toxic hepatitis in one patient. Further reasons for discontinuation of the study were: 4 additional patients with elevation of liver enzymes, 2 because of inefficacy and one unknown reason.

\section{Discussion}

In this 24-week, prospective, open-label trial with infliximab in 101 patients with active AS, given in daily practice in non-specialised rheumatological clinics and private practices, the quality of life in the majority of patients highly improved by therapy.

Measured by both the physical and the mental component of the SF-36, there was significant improvement at the final examination. This was similar to the results observed in the placebo controlled trial with infliximab (17) where also significant results for the physical and mental health after 24 weeks could be observed. In contrast, in the second placebo controlled trial with infliximab, the ASSERT study $(10,18)$, and the recent adalimumab trial in AS (19) there was no significant improvement for the mental component in the active groups after 24 weeks. Similarly, in a placebo controlled trial with etanercept (20) no significant improvement of the SF-36 was observed after 16 weeks of treatment, but there was efficacy for all subscales after 48 weeks. When compared with the age matched German database (21) the SF-36 for the physical health at baseline was highly impaired compared to the normal population (27.6 versus 51.0) in the present study but improved considerably (40.9). Interestingly in the present clinical trial patients started with a lower level for the mental component (44.4) than an age matched normal population (51.4), but after treatment the score for the mental component was even higher (53.0) after 28 weeks compared to the normal population (21).

With respect to effects on signs and symptoms, a BASDAI 50\% improvement at week 28 was reached by $66.3 \%$ of patients. This is better than that observed in the registration trials with infliximab, where a response in the infliximab group 
in $52.9 \%(7)$ and $51.0 \%(10,18)$ of patients was seen. This observation is concordant with another open label trial with 50 patients showing an even higher improvement after 3 infusions (22), with an ASAS 50 response at week 16 in $86 \%$ of patients. But in contrast to the present study, only patients with elevated CRP levels were included suggesting that these patients are prone to a higher response rate. We recently reported that a BASDAI 50\% response rate can be achieved in AS patients treated with TNF-blockers in $70 \%$ or more if selected for shorter disease duration, better function or elevated CRP $(23,24)$. Thus, these data suggest that a good or very good response can be expected in up to two-thirds of patients in daily clinical practice if the right patients are chosen for treatment.

It has been shown that the cost of AS increases with higher disease activity, functional impairment and reduced quality of life $(25,26)$. These factors also contribute to unemployment (3). In our study, work productivity improves significantly after 24 weeks of therapy with infliximab as shown by the single components of the WPAI-SHP score. Most of the patients were within an employable age between 18 and 60 but less patients were employed compared to AS patients with the same disease duration from the German Database (27). This is most probably due to higher disease activity and greater functional impairment in the AS patients included in our trial. The physical but not the mental component of the SF-36 was also significantly lower in unemployed in comparison to employed patients. Interestingly, there was a substantial increase in employment from $61.4 \%$ to $70 \%$ in patients before and after treatment. Among the group of patients who were employed at baseline there was a significantly higher improvement of BASDAI and BASFI compared to the group who were not employed at baseline indicating that employed patients might rank a similar level of efficacy higher. Also in the group of patients who had been employed at start of the study the missing hours at work during the past 7 days decreased by $50 \%$. It was reported before that after one year of therapy with infliximab there is a decrease in days of sick leave by more than $50 \%$ (28), which is in line with our results.

Our study confirms that infliximab given in a dose of $5 \mathrm{mg}$ per $\mathrm{kg}$ body weight has a rapid onset of action and efficacy. This seems to be faster compared to the other two TNF-blockers etanercept and adalimumab $(6,29)$ although no head to head study between the drugs has been performed until now. The most likely reasons for this difference between the drugs at the start of treatment are the intravenous route of administration and the higher loading dose in case of infliximab treatment.

Recent recommendations on the treatment of AS with TNF-blockers suggest to continue treatment after 12 weeks only if a BASDAI 50 improvement or a decrease of at least 2 points of the BASDAI 0 - 10 scale was achieved (30). In this subgroup of patients with a BASDAI 50 improvement after 12 weeks there was a dramatic drop in both the BASDAI, BASFI (Figs. 1a and 1b) and even the BASMI which would correspond to a considerable increase in the quality of life $(31,32)$. Because it is only recommended to treat these patients long term with TNF-blockers future socioeconomical analyses should preferentially concentrate on this group.

Taken into account the high costs for TNF-blocker treatment, it would be helpful if such a decision about continuation/ discontinuation of treatment were made even earlier. Here we show that only $6 \%$ of patients not reaching BASDAI 20 after 6 weeks of treatment with infliximab (thus after 2 infusions) will reach BASDAI 50 improvement after 12 weeks, suggesting a decision might be made that early for AS patients treated with infliximab. However, a BASDAI 50 improvement after 6 weeks cannot be used as a cut-off because $10 \%$ still reached BASDAI 50 after 12 weeks who did not reach this level of improvement after 6 weeks. Furthermore, in the present study we observed an increase in the number of patients reaching a BASDAI 50 response later than 12 weeks in only $6 \%$ at week 28 , supporting the current ASAS recommendations.
The number and pattern of adverse events and serious adverse events did not differ from those known from the placebo controlled trials $(7,10)$ indicating that therapy of AS patients with infliximab is also safe when given in non-specialised centres for biological therapy in AS.

\section{Acknowledgements}

We thank the 21 participating German rheumatological centres for taking part in this study.

\section{References}

1. LEHTINEN K: Mortality and causes of death in 398 patients admitted to hospital with ankylosing spondylitis. Ann Rheum Dis 1993; 52: 174-6.

2. BRAUN J, PINCUS T: Mortality, course of disease and prognosis of patients with ankylosing spondylitis. Clin Exp Rheumatol 2002; 20 (Suppl. 28): S16-22.

3. BOONEN A: Socioeconomic consequences of ankylosing spondylitis. Clin Exp Rheumatol 2002; 20 (Suppl. 28): S23-6.

4. HAIBEL H, BRANDT HC, SONG IH et al:: No efficacy of subcutaneous methotrexate in active ankylosing spondylitis - a 16 weeks open label trial. Ann Rheum Dis 2007; 66: 419-21.

5. ZOCHLING J, VAN DER HEIJDE D, BURGOSVARGAS R et al.: ASAS/EULAR recommendations for the management of ankylosing spondylitis. Ann Rheum Dis 2006; 65: 44252.

6. BRANDT J, KHARIOUZOV A, LISTING J et al Six-month results of a double-blind, placebo-controlled trial of etanercept treatment in patients with active ankylosing spondylitis. Arthritis Rheum 2003; 48: 1667-75.

7. BRAUN J, BRANDT J, LISTING J et al.:Treatment of active ankylosing spondylitis with infliximab: a randomised controlled multicentre trial. Lancet 2002; 359: 1187-93.

8. VAN DER HEIJDE D, KIVITZ A, SCHIFF MH et $a l .:$ Efficacy and safety of adalimumab in patients with ankylosing spondylitis: results of a multicenter, randomized, double-blind, placebo-controlled trial. Arthritis Rheum 2006; 54: 2136-46.

9. BRANDT J, HAIBEL H, CORNELY D et al.: Successful treatment of active ankylosing spondylitis with the anti-tumor necrosis factor alpha monoclonal antibody infliximab. Arthritis Rheum 2000; 43: 1346-52.

10. VAN DER HEIJDE D, DIJKMANS B, GEUSENS $\mathrm{P}$ et al.: Efficacy and safety of infliximab in patients with ankylosing spondylitis: results of a randomized, placebo-controlled trial (ASSERT). Arthritis Rheum 2005; 52: 58291.

11. VAN DER LINDEN S, VALKENBURG HA, CATS A: Evaluation of diagnostic criteria for ankylosing spondylitis. A proposal for modification of the New York criteria. Arthritis Rheum 1984; 27: 361-8.

12. VAN DER HEIJDE D, CALIN A, DOUGADOS M 
KHAN MA, VAN DER LINDEN S, BELLAMY N: Selection of instruments in the core set for DC-ART, SMARD, physical therapy, and clinical record keeping in ankylosing spondylitis. Progress report of the ASAS Working Group. Assessments in Ankylosing Spondylitis. J Rheumatol 1999; 26: 951-4.

13. WARE JE JR, SHERBOURNE CD: The MOS 36-item short-form health survey (SF-36). I. Conceptual framework and item selection. Med Care 1992; 30: 473-83.

14. VAN DER HEIJDE D, BELLAMY N, CALIN A, DOUGADOS M, KHAN MA, VAN DER LINDEN S: Preliminary core sets for endpoints in ankylosing spondylitis. Assessments in Ankylosing Spondylitis Working Group. J Rheumatol 1997; 24: 2225-9.

15. BRANDT J, LISTING J, SIEPER J, RUDWALEIT M, VAN DER HEIJDE D, BRAUN J: Development and preselection of criteria for shortterm improvement after anti-TNF $\alpha$ therapy in ankylosing spondylitis. Ann Rheum Dis 2004; 63: 1438-44.

16. REILLY MC, ZBROZEK AS, DUKES EM: The validity and reproducibility of a work productivity and activity impairment instrument. Pharmacoeconomics 1993; 4: 353-65.

17. BRAUN J, BRANDT J, LISTING J et al.: Longterm efficacy and safety of infliximab in the treatment of ankylosing spondylitis: an open, observational, extension study of a threemonth, randomized, placebo-controlled trial. Arthritis Rheum 2003; 48: 2224-33.

18. VAN DER HEIJDE D, DIJKMANS B, GEUSENS $\mathrm{P}$ et al.: Efficacy and safety of infliximab in patients with ankylosing spondylitis: results of a 24-week randomized, placebo-control- led trial (ASSERT). In: Annual meeting of the European League Against Rheumatism (EULAR); 2004; Berlin, Germany; 2004.

19. VAN DER HEIJDE D, LUO M, MATSUMOTO A et al.: Adalimumab Improves Health-Related Quality of Life in Patients with Active Ankylosing Spondylitis - The ATLAS Trial. In: ACR; 2005; San Diego: Arthritis Rheumatism; 2005. p. S211.

20. DAVIS JC, VAN DER HEIJDE D, DOUGADOS M, WOOLLEY JM: Reductions in health-related quality of life in patients with ankylosing spondylitis and improvements with etanercept therapy. Arthritis Rheum 2005; 53: 494501

21. BULLINGER MIK: SF-36 Fragebogen zum Gesundheitszustand. Göttingen: HogrefeVerlag; 1998.

22. BREBAN M, VIGNON E, CLAUDEPIERRE P et $a l .:$ Efficacy of infliximab in refractory ankylosing spondylitis: results of a six-month open-label study. Rheumatology (Oxford) 2002; 41: 1280-5.

23. RUDWALEIT M, LISTING J, BRANDT J, BRAUN J, SIEPER J: Prediction of a major clinical response (BASDAI 50) to tumour necrosis factor alpha blockers in ankylosing spondylitis. Ann Rheum Dis 2004; 63: 665-70.

24. RUDWALEIT M, LISTING J, BRANDT J, BRAUN J, SIEPER J: Prediction of a major clinical response (BASDAI 50) to TNF$\alpha$ blockers in ankylosing spondylitis. Ann Rheum Dis 2004; 63: 665-670.

25. WARD MM: Health-related quality of life in ankylosing spondylitis: a survey of 175 patients. Arthritis Care Res 1999; 12: 247-55.

26. ANTONI C, MAINI RN, GRUNKE N: Coopera- tive on quality of life in rheumatic disease: Results of a survey among 6000 patients across 11 European countries. Arthritis Rheum 2002; 46 (Suppl. 9): S76.

27. MAU W, LISTING J, HUSCHER D, ZEIDLER H, ZINK A: Employment across chronic inflammatory rheumatic diseases and comparison with the general population. J Rheumatol 2005; 32: 721-8.

28. LISTING J, BRANDT J, RUDWALEIT M, ZINK A, SIEPER J, BRAUN J: Impact of anti-tumour necrosis factor alpha treatment on admissions to hospital and days of sick leave in patients with ankylosing spondylitis. Ann Rheum Dis 2004; 63: 1670-2.

29. HAIBEL H, RUDWALEIT M, BRANDT HC et al.: Adalimumab reduces spinal symptoms in active ankylosing spondylitis: Clinical and magnetic resonance imaging results of a fifty-two-week open-label trial. Arthritis Rheum 2006; 54: 678-81.

30. BRAUN J, PHAM T, SIEPER J et al.: International ASAS consensus statement for the use of anti-tumour necrosis factor agents in patients with ankylosing spondylitis. Ann Rheum Dis 2003; 62: 817-24.

31. KOBELT G, ANDLIN-SOBOCKI P, BROPHY S, JONSSON L, CALIN A, BRAUN J: The burden of ankylosing spondylitis and the cost-effectiveness of treatment with infliximab (Remicade). Rheumatology (Oxford) 2004; 43: 1158-66.

32. KOBELT G, ANDLIN-SOBOCKI P, MAKSYMOWYCH WP: The cost-effectiveness of infliximab (Remicade) in the treatment of ankylosing spondylitis in Canada. J Rheumatol 2006; 33: 732-40. 


\subsection{Therapie der nichtröntgenologischen axialen SpA}

\subsubsection{Adalimumab bei nicht-röntgenologischer axialer Spondyloarthritis}

In einer eigenen weltweit ersten Placebo-kontrollierten Studie habe ich 46 Patienten mit nr-axSpA mit einer Therapie mit dem TNF-Blocker Adalimumab über zunächst 12 Wochen und einer anschließend offenen Verlängerung über weitere 40 Wochen und einer durchschnittlichen Krankheitsdauer von 8 Jahren untersucht [64]. Ein gutes klinisches Ansprechen, definiert als 40\% Verbesserung anhand der ASAS Kriterien erreichten 50\% der Patienten. Auch in anderen klinischen Outcome Parametern war eine deutliche und signifikante Verbesserung gegenüber Placebo nachweisbar. Nach Umstellung der Placebo Gruppe auf Adalimumab und Weiterbehandlung bis Woche 52 konnten signifikante Verbesserungen der Outcomeparameter auch in dieser Patientengruppe gezeigt werden.

In dieser Studie sprachen Patienten mit kurzer Krankheitsdauer deutlich besser an (Krankheitsdauer < 3 Jahre: ASAS 40 bei 80\% der Patienten) als Patienten mit längerer Krankheitsdauer (Krankheitsdauer > 3 Jahre: ASAS40 bei 17\% der Patienten). Weitere Prädiktoren für ein gutes klinisches Ansprechen in dieser Studie waren in einer multivariaten Regressionsuntersuchung junges Alter und erhöhtes CRP.

Das bessere Ansprechen von Patienten mit nr-axSpA und kurzer Krankheitsdauer wurde auch in anderen Studien bestätigt. In einer Placebo-kontrollierten Studie mit Infliximab, in der Patienten mit einer Krankheitsdauer von unter 3 Jahren, einem Anteil von Patienten mit nr-axSpA 87\%, positivem HLA-B27 und entzündlichen Veränderungen in der Baseline- MRT eingeschlossen wurden, erreichten nach 16 Wochen 55.6\% der Patienten die ASAS Kriterien für partielle Remission im Vergleich zur Placebo Gruppe, bei der dies nur in 10\% der Fälle erreicht wurde [65]. 
In einer anderen Studie, bei der Patienten mit kurzer Krankheitsdauer von unter 5 Jahren unabhängig vom Röntgenbild (51.3\% der Patienten erfüllten die modifizierten New York Kriterien für AS) entweder mit Etanercept oder Sulfasalazin behandelt wurden [66], erreichten nach 48 Wochen Therapie 50\% der mit Etanercept behandelten Patienten die ASAS Kriterien für partielle Remission im Vergleich zu 19\% der Patienten, die mit Sulfasalazin behandelt worden waren.

Insgesamt zeigen diese Daten, dass Patienten, unabhängig davon ob eine nr-axSpA oder eine AS vorliegt, ein gutes Ansprechen auf eine Therapie mit einem TNF-alpha Blocker erreichen. Patienten, die früh im Krankheitsverlauf mit einem TNF-Blocker behandelt werden, zeigen ein besseres klinisches Ansprechen. Insbesondere Patienten mit einer sehr kurzen Krankheitsdauer, unabhängig vom röntgenologischen Stadium der Erkrankung, haben eine sehr hohe Wahrscheinlichkeit, gut auf die Therapie anzusprechen.

Haibel H, Rudwaleit M, Listing J, Heldmann F, Wong RL, Kupper H, Braun J, Sieper J. Efficacy of Adalimumab in the Treatment of Axial Spondyloarthritis without Radiographically Defined Sacroiliitis, Arthritis Rheum. 2008 Jul;58(7):1981-91

http://dx.doi.org/10.1002/art.23606. 


\subsubsection{Langzeiteffektivität von Adalimumab bei Patienten mit nicht- röntgenologischer axialer Spondyloarthritis}

Nicht zuletzt wegen potentiell möglicher Nebenwirkungen und wegen der sehr hohen Kosten der Therapie mit TNF-alpha-Blockern stellte sich die Frage, ob bei gutem klinischen Ansprechen die Therapie beendet und ein anhaltender guter klinischer Status erhalten werden kann.

In der eigenen Studie mit Adalimumab bei nr-axSpA [64] wurde die Therapie bei allen Patienten in Woche 52 unterbrochen. Patienten, die ein gutes klinisches Ansprechen, definiert als Erreichen von ASAS40 in Woche 52, hatten, bekamen die Möglichkeit, bei Verschlechterung der Symptome erneut therapiert zu werden. Patienten, die kein gutes Ansprechen auf die Therapie hatten, beendeten die Studie in Woche 52 [67].

Von initial 46 in die Studie eingeschlossenen Patienten erreichten 23 ein gutes klinisches Ansprechen. In einem guten klinischen Zustand ohne erneute Therapie blieben 13\% der Patienten $(n=4)$. Die meisten Patienten mussten jedoch nach einem Schub der Krankheitsaktivität erneut mit Adalimumab behandelt werden (19/23 Patienten, 87\%) [67]. Die mittlere Dauer bis zum Wiederauftreten der Krankheitsaktivität betrug $14.7 \pm 5.5$ Wochen (Spannweite 3-27 Wochen). In einer multivariaten Regressionsanalyse fanden sich keine Prädiktoren für erneute Krankheitsaktivität und für die unterschiedliche Dauer bis zum Wiederauftreten von Aktivität.

Nach 1 Jahr Wiederbehandlung erreichten 12/19 (63,2\%) Patienten erneut ein gutes Ansprechen gemäß ASAS40 und erfüllten 9/19 (47.4\%) der Patienten die ASAS Kriterien für partielle Remission. Nach 2 Jahren Wiederbehandlung erreichten 73.7\% der Patienten ( $n=15)$ ASAS40 und 63.2\% der Patienten $(n=12)$ die ASAS Kriterien für partielle Remission. Dies zeigt, dass die Mehrheit der Patienten mit initial gutem 
Ansprechen, Therapieunterbrechung und Wiederbehandlung nach Auftreten eines Schubs ein erneutes gutes Ansprechen auf die Therapie erreichen.

In der oben schon erwähnten klinischen Studie mit Infliximab bei Patienten mit langjähriger AS [12] wurde nach 3 Jahren die Therapie unterbrochen. Bei nur einem Patienten musste die Therapie über einen Beobachtungszeitraum von 48 Wochen nicht wieder begonnen werden, 97.6\% der Patienten mussten nach einer durchschnittlichen Zeit bis zum Wiederauftreten der Krankheitsaktivität von 17.5 Wochen wieder behandelt werden [68]. Auch in einer Studie mit Etanercept mussten alle Patienten, die initial gut auf die Therapie angesprochen hatten, nach Therapieunterbrechung bei Wiederauftreten von Krankheitsaktivität innerhalb von einigen Wochen wieder behandelt werden [69].

In der ebenfalls bereits erwähnten englischen Studie mit Patienten mit axialer SpA und kurzer Krankheitsdauer von weniger als 3 Jahren (mittlere Krankheitsdauer 15 Monate), wurde Infliximab nach 12 Wochen Therapie unterbrochen. 17/19 Patienten (87\%), die ursprünglich mit Placebo behandelt worden waren, hatten weiterhin eine aktive Erkrankung, während dies nur bei 12/20 (60\%) der Patienten mit Infliximab der Fall war, $\mathrm{p}=0.035$. Die mediane Zeit bis zum Schub betrug 0 Wochen für Placebo- Patienten und 16 Wochen für die Infliximab Gruppe. 8/20 (40\%) der Patienten aus der Infliximab Gruppe verblieben in therapiefreiem gutem klinischem Zustand über den Beobachtungszeitraum von 40 Wochen [70]. Damit war dies die einzige Studie, die eine länger anhaltende Medikamentenfreie Remission bei Frühtherapie in einem höheren Prozentsatz der Patienten berichtet. Allerdings sind diese Ergebnisse bisher nicht vollständig publiziert. 
Insgesamt muss man daher bei Patienten mit axialer Spondyloarthritis von einer längerfristigen Therapie ausgehen.

Eigene Publikation: Haibel H, Heldmann F, Listing J, Kupper H, Braun J, Sieper J. Long term efficacy of adalimumab after drug withdrawal and retreatment of flare patients in active non-radiographic axial spondyloarthritis. Arthritis Rheum. 2013 Aug;65(8):22113

http://dx.doi.org/10.1002/art.38014 


\section{Diskussion:}

In der vorliegenden Arbeit habe ich verschiedene klinische Studien bei Patienten mit AS und nr-axSpA sowie eine Arbeit zur Evaluation des Lebensqualitätsfragebogens SF-12 im deutschen Sprachraum bei Patienten mit AS zusammengefasst. Hintergrund der Therapiestudien war, dass es zum Zeitpunkt der Durchführung der Studien neben NSAR keine etablierten medikamentösen Therapien für die AS gab. Für die nr-axSpA wurden erst kürzlich neue Klassifikationskriterien entwickelt [39] und so der Weg für Therapiestudien in einer gut charakterisierten Patientengruppe geebnet. In den letzten 15 Jahren ist ein entscheidender Durchbruch in der Therapie der AS mit TNF-alpha Blockern gelungen $[15,24,36,52]$. Jedoch sprechen nur ca. 50\% der Patienten gut auf eine solche Therapie an, es kann schwerwiegende Nebenwirkungen geben oder es können Kontraindikationen für den Einsatz von TNF-alpha Blockern bestehen, so dass die Suche nach anderen krankheitsmodifizierenden Medikamenten sehr wichtig ist. Zudem wird immer wieder die Frage diskutiert, ob in Parallelschluss zur Therapie der RA, eine Kombination mit TNF-alpha Blockern und krankheitsmodifizierenden Medikamenten zu einer besseren klinischen Wirksamkeit führen kann. Insofern ist die Suche nach etwaigen Kombinationspartnern bei Patienten mit AS interessant.

Der Lebensqualitätsfragebogen SF-36 [7] ist ein ubiquitär einsetzbares Instrument bei verschiedenen Krankheitsbildern und bei der Normalbevölkerung und macht somit die in einer bestimmten Gruppe gemessene Lebensqualität mit der anderer Gruppen vergleichbar. In der eigenen Untersuchung habe ich im Rahmen einer klinischen Studie [12] den kürzeren und schneller ausfüllbaren Fragebogen SF-12, der aus dem SF-36 extrahierbar ist, untersucht und gefunden, dass bei Patienten mit AS der SF-12 zur Erfassung der Lebensqualität in den Summenscores für körperliche und psychische Gesundheit vergleichbar gut zum SF-36 einsetzbar ist [43]. Es konnte in dieser 
Untersuchung auch gezeigt werden, dass Patienten mit aktiver AS gegenüber einer alters- und Geschlechts-gleichen „Normal“- Population aus der deutschen Normstichprobe eine deutlich eingeschränkte Lebensqualität insbesondere im Bereich der Funktionskapazität haben. Zudem konnte gezeigt werden, dass die kurz- und mittelfristige Therapie mit Infliximab zu einer deutlichen Verbesserung der Lebensqualität führt. So wurde der SF-12 in unserer Kohortenstudie für Patienten mit Frühformen von AS oder SpA erfolgreich und mit dem Vorteil der Zeitersparnis eingesetzt [71].

In einer offenen klinischen Studie mit 12 Patienten mit aktiver AS, die mit dem Bisphosphonat Pamidronat über insgesamt 6 Monate behandelt wurden, konnte kein deutlicher Effekt durch das Medikament auf die Krankheitsaktivität gefunden werden [32]. Wenn man die ASAS20 Response der Patienten mit einer Placebo Response von ca. 25\% aus der Originalarbeit zur Evaluation der ASAS-Response-Kriterien [72] oder einer Studie mit TNF-Blockern [12] vergleicht, so hatten die mit Pamidronat behandelten Patienten keine höhere Responserate. Zudem fanden wir in der Studie keine Verbesserung der peripheren Manifestationen. Im Gegensatz dazu berichtete Maksymowych et al. in 3 monozentrischen Studien aus Kanada [48-50], dass Pamidronat, effektiv bei NSAR-refraktärer AS mit axialer und peripherer Beteiligung die Krankheitsaktivität unterdrückt. In der ersten Studie wurden 16 Patienten mit einer mittleren Krankheitsdauer von 12.3 Jahren mit 6 monatlichen Infusionen behandelt, 8 Patienten erhielten $30 \mathrm{mg}$ Pamidronat über 3 Monate, gefolgt von 60mg/ Monat über weitere 3 Monate, 8 Patienten erhielten 60 mg Pamidronat über 3 Monate. Es fanden sich signifikante Verbesserungen in der Krankheitsaktivität (BASDAI), Beweglichkeit (BASMI) und Blutsenkungsgeschwindigkeit (BSG) insbesondere bei den Patienten, die 6 Monate behandelt wurden. Hier fand sich eine 38\% Reduktion des BASDAI nach 6 
Monaten [49]. In einer zweiten Untersuchung wurden 9 Patienten mit verschiedenen SpA-Formen (5 Patienten AS, 3 Patienten undifferenzierte SpA, 1 Patient reaktive Arthritis) und NSAR-refraktärer peripherer Arthritis eingeschlossen. Es wurden 60mg Pamidronat an den Tagen 1, 2, 14, 28 und 56 iv. verabreicht. Eine Verbesserung zeigte sich insbesondere bei Patienten, die mindestens 4 Infusionen erhalten hatten. Der mittlere BASDAI verbesserte sich um 44.2\%, der mittlere BASFI um 47.3\%, die mittlere Anzahl geschwollener Gelenke um 93.8\% und das CRP um 66.9\% [50]. In einer daraufhin durchgeführten kontrollierten doppelblinden Studie wurden insgesamt 84 Patienten entweder mit 60 mg oder 10 mg Pamidronat als aktiver Vergleich monatlich über 6 Monate behandelt [48]. Eine signifikante Verbesserung der Outcome Parameter war nicht nach 3 Monaten, jedoch nach 6 Monaten zu verzeichnen. Eine 25\%Verbesserung des BASDAI war bei $63 \%$ der Patienten in der 60 mg Gruppe im Vergleich zu 30.2\% der Patienten in der $10 \mathrm{mg}$ Gruppe eingetreten. Eine 50\% Besserung des BASDAl in der 60mg Gruppe fand sich bei 39\% der Patienten. Die Entzündungsparameter (CRP) änderten sich nicht. Da noch weitere im Verlauf publizierte klinische Studien gemeinsam mit der hier vorgestellten Arbeit insgesamt inkonklusive Ergebnisse erbrachten [30, 31, 44, 46-50], wurde die Anwendung von Pamidronat nicht in die Theapieempfehlungen der SpA aufgenommen [37].

Obwohl in der täglichen Praxis eine substantielle Gruppe von Patienten mit AS mit MTX behandelt wird, gibt es keine Beweise für dessen Effektivität bei AS [13, 17, 18]. Zudem waren die bis dato durchgeführten klinischen Studien entweder in zu niedriger Dosierung, mit zu geringen Patientenzahlen oder mit nicht evaluierten Outcomeparametern durchgeführt worden. In der hier vorgestellten klinischen Studie bei Patienten mit aktiver AS wurde im Gegensatz zu vorangegangen Studien eine Dosis gewählt, die an der Obergrenze der normalerweise bei RA verwendeten Dosierungen 
liegt (20mg s.c./ Woche). Die Therapie erfolgte über 16 Wochen mit negativem Ergebnis für den axialen Befall [55]. ASAS20 wurde hier bei 25\% der Patienten erreicht, was wiederum eine zu erwartenden Placebo Response, wie sie aus anderen Studien bekannt ist [12, 72], nicht übersteigt. Diese Erkenntnisse flossen ebenfalls in die Therapieempfehlungen der ASAS/ EULAR ein mit der Empfehlung, dass MTX bei Patienten mit axialem Befall nicht angewendet werden sollte [37]. Optional kann MTX für den peripheren Gelenkbefall eingesetzt werden, hierfür gab es Hinweise in meiner eigenen Arbeit, allerdings ohne Nachweis statistischer Signifikanz [55].

Bislang gab es keinerlei Studien zum Einsatz von Prednisolon per os bei Patienten mit AS [37]. In einer weltweit ersten Placebo- kontrollierten Studie untersuchte ich die Wirksamkeit von Prednisolon 20 mg versus 50 mg gegenüber Placebo bei Patienten mit NSAR-refraktärer AS über einen Zeitraum von 2 Wochen [56]. Es zeigte sich ein signifikanter Effekt für 50mg/Tag jedoch nicht für die Gruppe der mit 20 mg behandelten Patienten gegenüber Placebo. Das CRP reduzierte sich signifikant in beiden Therapiegruppen, während dies in der Placebo Gruppe nicht der Fall war. Der ASDAS, als Aktivitätsscore, der auch das CRP beinhaltet, sank ebenfalls signifikant in beiden Gruppen aber nicht in der Placebo Gruppe. Dies könnte als Überbrückungstherapie für Patienten mit Schub der Erkrankung sinnvoll sein, die z.B. nicht (oder noch nicht) Kandidaten für eine Therapie mit TNF-Blocker sind. Ob und wie lange der Therapieeffekt über den Therapiezeitraum hinaus zum Beispiel mit einer Erhaltungsdosis von $10 \mathrm{mg}$ Prednisolon bestehen bleibt, könnte Gegenstand einer weiteren Studie sein. Eine Langzeitanwendung von Prednisolon in höherer Dosierung wäre wegen des bekannten Nebenwirkungsprofils nicht zu akzeptieren.

Weitere Erkenntnisse zu schon vorbekannten klinischen Erfolgen gemessen mittels Krankheitsaktivität und Funktion von TNF-Blockern bei AS konnten bezüglich 
Lebensqualität, gemessen mittels SF-36, und in der täglichen Praxis in einer klinischen Studie mit dem TNF-Blocker Infliximab bei Patienten mit aktiver AS gewonnen werden [60]. Nach 24 Wochen Therapie mit Infliximab fand sich eine signifikante Verbesserung für die körperliche und psychische Komponente des Scores, ganz ähnlich wie in einer vorangegangenen Placebo-kontrollierten Studie mit demselben TNF-alpha Blocker [12]. Zudem konnte eine deutliche Verbesserung der Arbeitsproduktivität und täglichen Aktivität sowie eine deutliche Reduktion verpasster Arbeitsstunden, gemessen mittels WPAI, sowie der Anzahl der Fehlzeiten auf Arbeit um 50\% innerhalb des kurzen Studienzeitraums von 28 Wochen festgestellt werden. Diese Ergebnisse unterstützten die bisherigen Ergebnisse, dass der TNF-Blocker Infliximab ein gut wirksames Medikament in der Therapie der aktiven NSAR-refraktären AS ist. In aus Subgruppenanalysen gewonnenen Ergebnissen zur Prädiktion eines guten klinischen Ansprechens zeigte sich, dass eine Therapie früh im Krankheitsverlauf mit höheren Verbesserungen einhergeht.

Die nr-axSpA wurde in der vergangenen Dekade neu definiert [39]. Ich habe weltweit die erste doppelblinde Placebo-kontrollierte klinische Studie mit dem TNF-Blocker Adalimumab in dieser Patientengruppe durchgeführt [64]. Es konnte gezeigt werden, dass diese Patientengruppe ähnlich gut auf den TNF-Blocker anspricht wie Patienten mit etablierter AS. Die durchschnittliche Krankheitsdauer in der Studie betrug 8 Jahre. In Subgruppenanalysen habe ich gezeigt, dass insbesondere Patienten mit kurzer Krankheitsdauer aber auch Patienten mit erhöhtem CRP zu Baseline profitieren. Auf Basis der Erkenntnisse aus dieser Studie wurde eine große multizentrische Studie in der Patientengruppe angeschlossen [73] und Adalimumab erhielt als erster TNF-Blocker die Zulassung in der Indikation der nr-axSpA. 
Eine weitere Frage, die in der Studie aufgegriffen wurde, war, ob Patienten, die gut auf die Therapie angesprochen haben, ohne weitere Therapie in gutem klinischem Zustand verbleiben können [67]. Hierbei stellte sich heraus, dass die Mehrheit der Patienten mit nicht-röntgenologischer axialer SpA einer kontinuierlichen Therapie bedürfen und es nach Absetzen des Medikaments bei Patienten mit gutem Ansprechen im ersten Jahr der Studie nach einigen Wochen zum Krankheitsschub kommt. Nach Wiederaufnahme der Therapie spricht aber die Mehrheit der Patienten erneut gut und anhaltend auf Adalimumab an. 


\section{Zusammenfassung}

In der vergangenen Dekade haben sich neben der Erstellung neuer Klassifikationskriterien die Messinstrumente zur Evaluation der Erkrankung und die therapeutischen Optionen für die AS deutlich erweitert und neue Erkenntnisse aus klinischen Studien sind zu aktuellen Therapieempfehlungen zusammengefasst worden. In der vorliegenden Arbeit habe ich meinen Beitrag aus klinischen Studien bei Patienten mit AS und nr-axSpA zusammengefasst. In einer klinischen Studie mit Pamidronat konnte kein deutlicher Effekt des Medikaments auf die Besserung der Krankheitsaktivität gefunden werden [32]. Da sonstige klinische Studien gemeinsam mit der hier vorgestellten Arbeit inkonklusive Ergebnisse erbrachten [30, 31, 44, 46-50], wurde die Anwendung von Pamidronat nicht in die Theapieempfehlungen integriert [37]. Obwohl in der täglichen Praxis eine substantielle Gruppe von Patienten mit AS mit MTX behandelt wird, gibt es keine Beweise für dessen Effektivität bei dieser Erkrankung [13, 17, 18]. In der hier vorgestellten klinischen Studie wurde im Gegensatz zu vorangegangen Studien eine ausreichende Dosis (20mg s.c.) über 16 Wochen mit negativem Ergebnis für den axialen Befall verabreicht [55]. Diese Erkenntnisse flossen ebenfalls in die Therapieempfehlungen der ASAS/ EULAR ein [37]. Optional kann MTX für den peripheren Gelenkbefall eingesetzt werden, hierfür gab es Hinweise in meiner eigenen Arbeit, allerdings ohne Nachweis statistischer Signifikanz [55].

Bislang gab es keinerlei Studien zum Einsatz von Prednisolon per os bei Patienten mit AS [37]: In einer weltweit ersten Placebo- kontrollierten Studie untersuchte ich die Wirksamkeit von Prednisolon 20 mg versus 50 mg gegenüber Placebo bei Patienten mit NSAR-refraktärer AS über einen Zeitraum von 2 Wochen [56]. Es zeigte sich ein signifikanter Effekt für 50mg/Tag gegenüber Placebo. Dies könnte sinnvoll als 
Überbrückungstherapie für Patienten mit Schub der Erkrankung sein, die z.B. nicht (oder noch nicht) Kandidaten für eine Therapie mit TNF-Blocker sind.

Weitere Erkenntnisse zu schon vorbekannten klinischen Erfolgen gemessen mittels Krankheitsaktivität und Funktion von TNF-Blockern bei AS konnten bezüglich Lebensqualität, gemessen mittels SF-36, und in der täglichen Praxis gewonnen werden [60]. Zudem konnte eine deutliche Verbesserung der Arbeitsproduktivität und täglichen Aktivität sowie eine deutliche Reduktion verpasster Arbeitsstunden, gemessen mittels WPAI, innerhalb des kurzen Studienzeitraums von 28 Wochen festgestellt werden. Diese Ergebnisse unterstützten die bisherigen Ergebnisse, dass der TNF-Blocker Infliximab ein gut wirksames Medikament in der Therapie der aktiven NSAR-refraktären AS ist.

In aus Subgruppenanalysen gewonnenen Ergebnissen zur Prädiktion eines guten klinischen Ansprechens zeigte sich, dass eine Therapie früh im Krankheitsverlauf mit höheren Verbesserungen einhergeht. Die nr-axSpA wurde in der vergangenen Dekade neu definiert [39]. Eine klinische Studie mit dem TNF-Blocker Adalimumab wurde in dieser Patientengruppe weltweit erstmals in einer klinischen doppelblinden Placebokontrollierten Studie untersucht [64]. Hierbei konnte gezeigt werden, dass diese Patientengruppe ähnlich gut auf den TNF-Blocker anspricht wie Patienten mit AS. In Subgruppenanalysen konnte gezeigt werden, dass insbesondere Patienten mit kurzem Krankheitsverlauf profitieren. Auf Basis dieser Erkenntnisse wurde eine große multizentrische Studie in der Patientengruppe angeschlossen [73] und Adalimumab erhielt als erster TNF-Blocker die Zulassung in dieser Indikation. Eine weitere Frage, die in der Studie aufgegriffen wurde, war, ob Patienten, die gut auf die Therapie angesprochen haben, ohne weitere Therapie in gutem klinischem Zustand verbleiben können [67]. Hierbei stellte sich heraus, dass die Mehrheit der Patienten mit nicht- 
röntgenologischer axialer SpA einer kontinuierlichen Therapie bedürfen und es nach Absetzen des Medikaments nach einigen Wochen zum Krankheitsschub kommt. Nach Wiederaufnahme der Therapie spricht wiederum die Mehrheit der Patienten erneut gut an. 


\section{Literaturverzeichnis}

1. Braun, J., et al., Prevalence of spondylarthropathies in HLA-B27 positive and negative blood donors. Arthritis Rheum, 1998. 41(1): p. 58-67.

2. Gran, J.T., G. Husby, and M. Hordvik, Prevalence of ankylosing spondylitis in males and females in a young middle-aged population of Tromso, northern Norway. Ann Rheum Dis, 1985. 44(6): p. 359-67.

3. Feldtkeller, E., [Age at disease onset and delayed diagnosis of spondyloarthropathies]. Z Rheumatol, 1999. 58(1): p. 21-30.

4. van der Linden, S., H.A. Valkenburg, and A. Cats, Evaluation of diagnostic criteria for ankylosing spondylitis. A proposal for modification of the New York criteria. Arthritis Rheum, 1984. 27(4): p. 361-8.

5. van der Heijde, D., et al., Preliminary core sets for endpoints in ankylosing spondylitis. Assessments in Ankylosing Spondylitis Working Group. J Rheumatol, 1997. 24(11): p. 2225-9.

6. van der Heijde, D., et al., ASsessment in Ankylosing Spondylitis International Working Group/Spondylitis Association of America recommendations for conducting clinical trials in ankylosing spondylitis. Arthritis Rheum, 2005. 52(2): p. 386-94.

7. Medical Outcome Trust. How to score the SF-36 Health Survey, 1994, Medical Outcome Trust: Boston.

8. van der Heijde, D., et al., ASDAS, a highly discriminatory ASAS-endorsed disease activity score in patients with ankylosing spondylitis. Ann Rheum Dis, 2009. 68(12): p. 1811-8.

9. Dougados, M., et al., Conventional treatments for ankylosing spondylitis. Ann Rheum Dis, 2002. 61 Suppl 3: p. iii40-50.

10. Braun, J., et al., Nested polymerase chain reaction strategy simultaneously targeting DNA sequences of multiple bacterial species in inflammatory joint diseases. II. Examination of sacroiliac and knee joint biopsies of patients with spondyloarthropathies and other arthritides. J Rheumatol, 1997. 24(6): p. 1101-5.

11. Brandt, J., et al., Successful treatment of active ankylosing spondylitis with the anti-tumor necrosis factor alpha monoclonal antibody infliximab. Arthritis Rheum, 2000. 43(6): p. 1346-52.

12. Braun, J., et al., Treatment of active ankylosing spondylitis with infliximab: a randomised controlled multicentre trial. Lancet, 2002. 359(9313): p. 1187-93.

13. Chen, J. and C. Liu, Methotrexate for ankylosing spondylitis. Cochrane Database Syst Rev, 2004(3): p. CD004524.

14. Chen, J. and C. Liu, Sulfasalazine for ankylosing spondylitis. Cochrane Database Syst Rev, 2005(2): p. CD004800.

15. Dougados, M., et al., Sulfasalazine in the treatment of spondylarthropathy. A randomized, multicenter, double-blind, placebo-controlled study. Arthritis Rheum, 1995. 38(5): p. 618-27. 
16. Braun, J., et al., Efficacy of sulfasalazine in patients with inflammatory back pain due to undifferentiated spondyloarthritis and early ankylosing spondylitis: a multicentre randomised controlled trial. Ann Rheum Dis, 2006. 65(9): p. 1147-53.

17. Chen, J., C. Liu, and J. Lin, Methotrexate for ankylosing spondylitis. Cochrane Database Syst Rev, 2006(4): p. CD004524.

18. Chen, J., et al., Methotrexate for ankylosing spondylitis. Cochrane Database Syst Rev, 2013. 2: p. CD004524.

19. Altan, L., et al., Clinical investigation of methotrexate in the treatment of ankylosing spondylitis. Scand J Rheumatol, 2001. 30(5): p. 255-9.

20. Gonzalez-Lopez, L., et al., Efficacy of methotrexate in ankylosing spondylitis: a randomized, double blind, placebo controlled trial. J Rheumatol, 2004. 31(8): p. 1568-74.

21. Roychowdhury, B., et al., Methotrexate in severe ankylosing spondylitis - a randomized placebo-controlled study. Rheumatology, 2001. 40(Suppl1: 43).

22. Sampaio-Barros, P.D., et al., Methotrexate in the treatment of ankylosing spondylitis. Scand J Rheumatol, 2000. 29(3): p. 160-2.

23. Braun, J., et al., Computed tomography guided corticosteroid injection of the sacroiliac joint in patients with spondyloarthropathy with sacroiliitis: clinical outcome and followup by dynamic magnetic resonance imaging. J Rheumatol, 1996. 23(4): p. 659-64.

24. Mintz, G., et al., Intravenous methylprednisolone pulse therapy in severe ankylosing spondylitis. Arthritis Rheum, 1981. 24(5): p. 734-6.

25. Peters, N.D. and L. Ejstrup, Intravenous methylprednisolone pulse therapy in ankylosing spondylitis. Scand J Rheumatol, 1992. 21(3): p. 134-8.

26. Richter, M.B., et al., The effects of intravenous pulse methylprednisolone on immunological and inflammatory processes in ankylosing spondylitis. Clin Exp Immunol, 1983. 53(1): p. 51-9.

27. Rumiantseva, O.A., et al., [Intravenous high dose glucocorticoids in patients with ankylosing spondylitis]. Ter Arkh, 2006. 78(12): p. 71-5.

28. van der Heijde, D., et al., 2010 Update of the international ASAS recommendations for the use of anti-TNF agents in patients with axial spondyloarthritis. Ann Rheum Dis, 2011. 70(6): p. 905-8.

29. Van Offel, J.F., et al., Influence of cyclic intravenous pamidronate on proinflammatory monocytic cytokine profiles and bone density in rheumatoid arthritis treated with low dose prednisolone and methotrexate. Clin Exp Rheumatol, 2001. 19(1): p. 13-20.

30. Pennanen, N., et al., Effect of liposomal and free bisphosphonates on the IL-1 beta, IL-6 and TNF alpha secretion from RAW 264 cells in vitro. Pharm Res, 1995. 12(6): p. 916-22.

31. Santra, G., et al., Assessment of the efficacy of pamidronate in ankylosing spondylitis: an open prospective trial. Singapore Med J, 2010. 51(11): p. 883-7.

32. Haibel, H., et al., Treatment of active ankylosing spondylitis with pamidronate. Rheumatology (Oxford), 2003. 42(8): p. 1018-20. 
33. Baraliakos X, L.J., Fritz C, Alten R, Burmester G, Krause A, Schewe S, Schneider M, Soerensen H, Zeidler H, Rudwaleit M, Sieper J, Braun J, Persistent Clinical Efficacy And Safety Of Inlifiximab In Patients With Ankylosing Spondylitis Over 7 Years - Evidence For Different Response To Anti-tnf-a Therapy. Arthritis and Rheumatism, 2008. 58(9 (Suppl)): p. S583.

34. Brandt, J., et al., Successful short term treatment of severe undifferentiated spondyloarthropathy with the anti-tumor necrosis factor-alpha monoclonal antibody infliximab. J Rheumatol, 2002. 29(1): p. 118-22.

35. Davis, J.C., Jr., et al., Recombinant human tumor necrosis factor receptor (etanercept) for treating ankylosing spondylitis: a randomized, controlled trial. Arthritis Rheum, 2003. 48(11): p. 3230-6.

36. van der Heijde, D., et al., Efficacy and safety of adalimumab in patients with ankylosing spondylitis: results of a multicenter, randomized, double-blind, placebo-controlled trial. Arthritis Rheum, 2006. 54(7): p. 2136-46.

37. Zochling, J., et al., ASAS/EULAR recommendations for the management of ankylosing spondylitis. Ann Rheum Dis, 2006. 65(4): p. 442-52.

38. Rudwaleit, M., M.A. Khan, and J. Sieper, The challenge of diagnosis and classification in early ankylosing spondylitis: do we need new criteria? Arthritis Rheum, 2005. 52(4): p. 1000-8.

39. Rudwaleit, M., et al., The development of Assessment of SpondyloArthritis international Society classification criteria for axial spondyloarthritis (part I): classification of paper patients by expert opinion including uncertainty appraisal. Ann Rheum Dis, 2009. 68(6): p. 770-6.

40. Sieper, J., et al., New criteria for inflammatory back pain in patients with chronic back pain: a real patient exercise by experts from the Assessment of SpondyloArthritis international Society (ASAS). Ann Rheum Dis, 2009. 68(6): p. 784-8.

41. Rudwaleit, M., et al., The development of Assessment of SpondyloArthritis international Society classification criteria for axial spondyloarthritis (part II): validation and final selection. Ann Rheum Dis, 2009. 68(6): p. 777-83.

42. Rudwaleit, M., et al., MRI in predicting a major clinical response to anti-tumour necrosis factor treatment in ankylosing spondylitis. Ann Rheum Dis, 2008. 67(9): p. 1276-81.

43. Haibel, $\mathrm{H}$., et al., [Measurement of quality of life in patients with active ankylosing spondylitis being treated with infliximab-a comparison of SF-36 and SF-12]. Z Rheumatol, 2004. 63(5): p. 393-401.

44. Cairns, A.P., et al., An open study of pulse pamidronate treatment in severe ankylosing spondylitis, and its effect on biochemical markers of bone turnover. Ann Rheum Dis, 2005. 64(2): p. 338-9.

45. Dougados, M., et al., The European Spondylarthropathy Study Group preliminary criteria for the classification of spondylarthropathy. Arthritis Rheum, 1991. 34(10): p. 1218-27.

46. Toussirot, E., et al., Transient efficacy of pulse pamidronate treatment in active spondylarthropathies: an open study of 35 cases. Clin Exp Rheumatol, 2006. 24(3): p. 348. 
47. Grover, R., et al., Treatment of ankylosing spondylitis with pamidronate: an open label study. Ann Rheum Dis, 2006. 65(5): p. 688-9.

48. Maksymowych, W.P., et al., A six-month randomized, controlled, double-blind, dose-response comparison of intravenous pamidronate (60 mg versus $10 \mathrm{mg}$ ) in the treatment of nonsteroidal antiinflammatory drug-refractory ankylosing spondylitis. Arthritis Rheum, 2002. 46(3): p. 766-73.

49. Maksymowych, W.P., et al., An open study of pamidronate in the treatment of refractory ankylosing spondylitis. J Rheumatol, 1998. 25(4): p. 714-7.

50. Maksymowych, W.P., et al., Clinical and radiological amelioration of refractory peripheral spondyloarthritis by pulse intravenous pamidronate therapy. $\mathrm{J}$ Rheumatol, 2001. 28(1): p. 144-55.

51. Ward, M.M. and S. Kuzis, Medication toxicity among patients with ankylosing spondylitis. Arthritis Rheum, 2002. 47(3): p. 234-41.

52. Inman, R.D., et al., Efficacy and safety of golimumab in patients with ankylosing spondylitis: results of a randomized, double-blind, placebo-controlled, phase III trial. Arthritis Rheum, 2008. 58(11): p. 3402-12.

53. Landewe, R., et al., Efficacy of certolizumab pegol on signs and symptoms of axial spondyloarthritis including ankylosing spondylitis: 24-week results of a double-blind randomised placebo-controlled Phase 3 study. Ann Rheum Dis, 2014. 73(1): p. 39-47.

54. Smolen, J.S., et al., EULAR recommendations for the management of rheumatoid arthritis with synthetic and biological disease-modifying antirheumatic drugs: 2013 update. Ann Rheum Dis, 2014. 73(3): p. 492-509.

55. Haibel, H., et al., No efficacy of subcutaneous methotrexate in active ankylosing spondylitis: a 16-week open-label trial. Ann Rheum Dis, 2007. 66(3): p. 419-21.

56. Haibel, H., et al., Efficacy of oral prednisolone in active ankylosing spondylitis: results of a double-blind, randomised, placebo-controlled short-term trial. Ann Rheum Dis, 2014. 73(1): p. 243-6.

57. Braun, J., et al., [Recommendations for the management of ankylosing spodylitis after ASAS/EULAR. Evaluation in the German language area]. Z Rheumatol, 2006. 65(8): p. 728-42.

58. Baraliakos, X., et al., Long-term outcome of patients with active ankylosing spondylitis with etanercept-sustained efficacy and safety after seven years. Arthritis Res Ther, 2013. 15(3): p. R67.

59. van der Heijde, D.M., et al., Physical function, disease activity, and health-related quality-of-life outcomes after 3 years of adalimumab treatment in patients with ankylosing spondylitis. Arthritis Res Ther, 2009. 11(4): p. R124.

60. Haibel, H., et al., Multicenter open-label study with infliximab in active ankylosing spondylitis over 28 weeks in daily practice. Clin Exp Rheumatol, 2008. 26(2): p. 247-52.

61. Braun, J., et al., Long-term efficacy and safety of infliximab in the treatment of ankylosing spondylitis: an open, observational, extension study of a three-month, randomized, placebo-controlled trial. Arthritis Rheum, 2003. 48(8): p. 2224-33. 
62. van der Heijde, D., et al., Efficacy and safety of infliximab in patients with ankylosing spondylitis: results of a randomized, placebo-controlled trial (ASSERT). Arthritis Rheum, 2005. 52(2): p. 582-91.

63. Breban, M., et al., Efficacy of infliximab in refractory ankylosing spondylitis: results of a six-month open-label study. Rheumatology (Oxford), 2002. 41(11): p. 1280-5.

64. Haibel, H., et al., Efficacy of adalimumab in the treatment of axial spondylarthritis without radiographically defined sacroiliitis: results of a twelve-week randomized, double-blind, placebo-controlled trial followed by an open-label extension up to week fifty-two. Arthritis Rheum, 2008. 58(7): p. 1981-91.

65. Barkham, N., et al., Clinical and imaging efficacy of infliximab in HLA-B27Positive patients with magnetic resonance imaging-determined early sacroiliitis. Arthritis Rheum, 2009. 60(4): p. 946-54.

66. Song, I.H., et al., Effects of etanercept versus sulfasalazine in early axial spondyloarthritis on active inflammatory lesions as detected by whole-body MRI (ESTHER): a 48-week randomised controlled trial. Ann Rheum Dis, 2011. 70(4): p. 590-6.

67. Haibel, $\mathrm{H}$., et al., Long-term efficacy of adalimumab after drug withdrawal and retreatment in patients with active non-radiographically evident axial spondyloarthritis who experience a flare. Arthritis Rheum, 2013. 65(8): p. 2211-3.

68. Baraliakos, X., et al., Clinical response to discontinuation of anti-TNF therapy in patients with ankylosing spondylitis after 3 years of continuous treatment with infliximab. Arthritis Res Ther, 2005. 7(3): p. R439-44.

69. Brandt, J., et al., Long-term efficacy and safety of etanercept after readministration in patients with active ankylosing spondylitis. Rheumatology (Oxford), 2005. 44(3): p. 342-8.

70. Barkham, N., et al., Clinical response and time to active disease following infliximab therapy in patients with HLA-B27 positive very early ankylosing spondylitis. . Ann Rheum Dis, 2009;. 68(Suppl3): p. 72.

71. Rudwaleit, M., et al., The early disease stage in axial spondylarthritis: results from the German Spondyloarthritis Inception Cohort. Arthritis Rheum, 2009. 60(3): p. 717-27.

72. Anderson, J.J., et al., Ankylosing spondylitis assessment group preliminary definition of short-term improvement in ankylosing spondylitis. Arthritis Rheum, 2001. 44(8): p. 1876-86.

73. Sieper, J., et al., Efficacy and safety of adalimumab in patients with nonradiographic axial spondyloarthritis: results of a randomised placebo-controlled trial (ABILITY-1). Ann Rheum Dis, 2013. 72(6): p. 815-22. 


\section{Danksagung}

Insbesondere möchte ich mich bei Prof. J. Sieper bedanken, der es mir ermöglichte, eine ausgezeichnete Ausbildung und genügend Freiraum für wissenschaftliche Arbeit zu bekommen. Unter seiner Aufsicht habe ich klinische Studien konzipiert, durchgeführt, ausgewertet und publiziert und konnte in jeder Hinsicht auf eine produktive, zuverlässige und lehrreiche Zusammenarbeit bauen. Nichts wäre möglich gewesen ohne das Team in der Rheumatologie. Hierbei möchte ich Prof. Dr. M. Rudwaleit, PD Dr. J. Brandt-Jürgens, Dr. K. Karberg, PD H. Appel, PD U. Syrbe, PD D. Poddubnyy, Dr. H. Brandt, I. Spiller und Dr. S. Hermann danken wie auch den Studienschwestern, von Anfang an B. Buß, im Verlauf N. Kempa, M. Erkelenz, L.Goldschmidt, R. Lies, R. Pauli, A. Langdon, B. Böttcher-Peters, A. Guischard, C. Dörwald und A. Drehmel.

Besonders bedanken möchte ich mich bei Dr. J. Listing aus dem Deutschen Rheumaforschungszentrem; der als kompetenter Ansprechpartner für alle statistischen Fragestellungen und Auswertungen zur Verfügung stand.

Die von uns durchgeführten klinischen Studien wären nicht ohne Unterstützung der mit uns kooperierenden Radiologen PD Dr. Kay-Geert Hermann und PD Z. Grozdanovic zustande gekommen.

Einige klinische Studien wurden multizentrisch durchgeführt. Hierbei danke ich insbesondere dem Studienteam in Herne um Prof. J. Braun, Dr. F. Heldmann, Dr. X. Baraliakos und Frau D. Krinitzki.

Für organisatorische Belange standen Frau Adelheid Ditten und Petra Rudwaleit stets kompetent zur Seite.

Neben Prof. J. Sieper und Prof. Dr. M. Rudwaleit haben Professor M. Zeitz/ Prof. B. Siegmund als Abteilungsleiter und Prof. T. Schneider als Leiter der Infektiologie meine klinische Ausbildung entscheidend geprägt. 
Nicht zuletzt wäre meine wissenschaftliche Forschungstätigkeit nicht möglich gewesen ohne die Unterstützung meiner Familie. 


\section{Erklärung}

$\S 4$ Abs. 3 (k) der HabOMed der Charité Berlin

Hiermit erkläre ich, dass

- weder früher noch gleichzeitig ein Habilitationsverfahren durchgeführt oder angemeldet wird bzw. wurde,

- welchen Ausgang ein durchgeführtes Habilitationsverfahren hatte,

- die vorgelegte Habilitationsschrift ohne fremde Hilfe verfasst, die beschriebenen Ergebnisse selbst gewonnen sowie die verwendeten Hilfsmittel, die Zusammenarbeit mit anderen Wissenschaftlern/ Wissenschaftlerinnen und mit technischen Hilfskräften sowie die verwendete Literatur vollständig in der Habilitationsschrift angegeben wurden

- Mir die geltende Habilitationsordnung bekannt ist. 